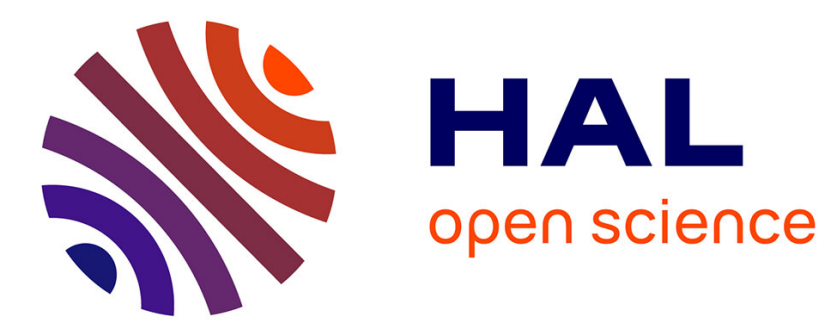

\title{
A Luenberger observer for reaction-diffusion models with front position data
}

Annabelle Collin, Dominique Chapelle, Philippe Moireau

\section{To cite this version:}

Annabelle Collin, Dominique Chapelle, Philippe Moireau. A Luenberger observer for reactiondiffusion models with front position data. Journal of Computational Physics, 2015, 300, pp.20. 10.1016/j.jcp.2015.07.044 . hal-01111675v2

\section{HAL Id: hal-01111675 \\ https://hal.inria.fr/hal-01111675v2}

Submitted on 13 Aug 2015

HAL is a multi-disciplinary open access archive for the deposit and dissemination of scientific research documents, whether they are published or not. The documents may come from teaching and research institutions in France or abroad, or from public or private research centers.
L'archive ouverte pluridisciplinaire HAL, est destinée au dépôt et à la diffusion de documents scientifiques de niveau recherche, publiés ou non, émanant des établissements d'enseignement et de recherche français ou étrangers, des laboratoires publics ou privés. 


\title{
A Luenberger observer for reaction-diffusion models with front position data
}

\author{
Annabelle Collin, Dominique Chapelle, Philippe Moireau \\ Inria Saclay Ile-de-France, Palaiseau, France
}

\begin{abstract}
We propose a Luenberger observer for reaction-diffusion models with propagating front features, and for data associated with the location of the front over time. Such models are considered in various application fields, such as electrophysiology, wild-land fire propagation and tumor growth modeling. Drawing our inspiration from image processing methods, we start by proposing an observer for the eikonal-curvature equation that can be derived from the reaction-diffusion model by an asymptotic expansion. We then carry over this observer to the underlying reaction-diffusion equation by an "inverse asymptotic analysis", and we show that the associated correction in the dynamics has a stabilizing effect for the linearized estimation error. We also discuss the extension to joint stateparameter estimation by using the earlier-proposed ROUKF strategy. We then illustrate and assess our proposed observer method with test problems pertaining to electrophysiology modeling, including with a realistic model of cardiac atria. Our numerical trials show that state estimation is directly very effective with the proposed Luenberger observer, while specific strategies are needed to accurately perform parameter estimation - as is usual with Kalman filtering used in a nonlinear setting - and we demonstrate two such successful strategies.
\end{abstract}

\section{Introduction}

Reaction-diffusion equations are considered in various application fields for modeling traveling wave propagation phenomena $[51,16]$. For instance, the electrical depolarization propagation is modeled in cardiac electrophysiology with the bidomain model and its approximation given by the monodomain model [83, 76, 49, 27]. In wild-land fire propagation, the wildfire spread is modeled by an advection-reaction-diffusion model [4]. Moreover, reaction-diffusion models exhibiting traveling wave solutions are also used in chemotaxis, with for example the Keller-Segel model [46], or for tumor-growth modeling, see $[82,38]$. When employing these models in practical applications, as for all natural and physical systems, a great difficulty consists in dealing with the many uncertain quantities that must be prescribed for running model simulations. These quantities include initial conditions and physical parameters of the model, which are difficult to measure. Fortunately, additional information is provided by available measurements, and these can be used to circumvent the uncertainties associated with the dynamical model definition. One typical example of available data lies in the propagation maps providing the location of the front at successive times, which can be measured using imaging modalities, 
in particular. For instance, in electrophysiology, Electrocardiographic Imaging (ECGI) aims at reconstructing cardiac potential isochrones [70]. In wildfire propagation, satellite images are processed to follow the wildfire front [73], whereas reconstructions of the tumor-growth area from medical images are now well performed, e.g. with MRI-imaging [41] sometimes combined with PET images [87].

Reducing the uncertainties and also estimating uncertain quantities - as for example the model parameters - for dynamical models by exploiting the available data is the very objective of data assimilation. The discipline of data assimilation historically arose in the context of geophysics - e.g. in oceanography and weather forecasting $[11,66]$ - with nowadays an increased focus on new application fields. As examples of these, we can cite the works of $[58,74]$ for data assimilation in wildfire propagation, of $[44,24]$ in tumorgrowth modeling, and also of $[52,12,30]$ in cardiac electrophysiology. In some of these applications, front isochrones are already used for data assimilation purposes, albeit at the cost of simplifying the original physical model to focus on front propagation, based on so-called eikonal equations [52] or level-set formulation [74], or by using a reduced-order modeling strategy [24]. Moreover, the question of finding the most effective measure of similarity between the observed and computed fronts remains a challenge, and this often leads to heuristic comparison strategies. Our objective in this paper is to propose a data assimilation strategy that can be applied to a wide variety of reaction-diffusion problems where front propagation is measured, without simplifying the original physical contents of the model, but benefitting from the maximum amount of information contained in the data at our disposal.

To reach this goal, we choose to rely on a sequential approach where the original dynamics is corrected at each time by a feedback control involving a discrepancy computed between the actual data and synthetic data reconstructed from the simulated trajectory. This family of methods comprises both the classical Kalman-based filtering approaches in which the feedback is computed from some kind of Riccati equation solution, and a Luenberger filtering approach [57] - popularized in data assimilation under the nudging terminology as initiated in $[1,45]$ - see [53] for a historical perspective - by which the feedback is designed to primarily seek a stabilization property of the feedback law, see various examples for infinite dimensional systems in $[5,71,56,19,13]$. Sequential approaches - also known as observer theory in the deterministic context - have to be compared with the maybe more popular variational approach of the so-called 4D-Var method [54] where, in essence, we minimize a - usually least-square based - cost function integrating during a period of time a compromise between (1) some a priori on the model and initial conditions and (2) the observation discrepancy between the actual measurements and the synthetic data produced by the model, see [64] for an example of variational approach in electrophysiology, or [44] in cancer growth. In the present article, we propose to formulate a joint state and parameter observer, where a Luenberger observer corrects the state, and an reduced-order optimal Kalman-based filter is restricted to the parametric space. This strategy - initiated in [62] for models based on partial differential equations - allows to perform the estimation with reasonable computing times since the Luenberger filter will not suffer from a "curse of dimensionality" arising from the discretization of the model, and the parameters to be identified are assumed to be smooth enough to be discretized with a limited number of degrees of freedom, compatible with an optimal Kalman-based approach. The parameter identification is a direct application of the original work of [61] which, however, requires that an adequate Luen- 
berger strategy has been previously designed to control the trajectory. Hence, the main originality of the present work is in formulating an effective Luenberger state observer for reaction-diffusion problems based on available front propagation data.

The outline of this paper is as follows. We first present in Section 2 the reactiondiffusion model and the type of data that we want to assimilate. Section 3 then concerns the state estimator. We start with the presentation of an observer suitable for a reactiondiffusion model with front propagation data. Then, we give the origin of the state estimator, starting with the presentation of an asymptotic level set formulation known as the eikonal-curvature equation. Drawing our inspiration from image processing techniques $[90,17,67]$, we present an observer valid for the eikonal-curvature equation, and we carry over the observer to the initial reaction-diffusion model by an inverse asymptotic analysis. The last - but not least - part of this section gives a mathematically-justified study of the observer model, and we prove that the correction term stabilizes the model of the error. The third section concerns the parameter estimation where the Reduced-Order Unscented Kalman Filtering (ROUKF) [61,60] is used. In the last section, we give several numerical illustrations of the proposed observer applied to cardiac electrophysiology. We present numerical simulations in 1D, but also realistic cases of electrophysiological depolarization propagation in the cardiac atria. These simulations validate, in a first part, the very effective state estimator. In a second part, using a joint state-parameter strategy, we identify some conductivity parameters.

\section{Problem setting}

We consider the following type of so-called reaction-diffusion model

$$
\left\{\begin{array}{rlcl}
\partial_{t} u-\vec{\nabla} \cdot(\overrightarrow{\vec{\sigma}} \cdot \vec{\nabla} u) & =k f(u), & \mathcal{B} \times(0, T), \\
(\overrightarrow{\vec{\sigma}} \cdot \vec{\nabla} u) \cdot \vec{n} & =0, & \partial \mathcal{B} \times(0, T), \\
u(\vec{x}, 0) & =u_{0}(\vec{x}), & \mathcal{B},
\end{array}\right.
$$

where $u$ is the (scalar) unknown field defined over a spatial domain $\mathcal{B}, \vec{\sigma}$ the diffusion tensor, $f(u)$ a reaction term and $k$ a modeling coefficient. For certain choices of nonlinear expressions of the function $f(u)$ and with adequate initial conditions, the solution $u$ exhibits some interesting propagative features, with a rapid transition occurring between different values around a traveling front $[72,48,88,55]$. This is the type of behavior considered in this paper, and we assume that we can accordingly benefit from data concerning the location of this front over time, for estimation purposes. Our objective will be to design an observer based on such measurements for the above model, typically in order to compensate for uncertainties in initial conditions and/or in modeling parameters.

We assume that the traveling front $\Gamma_{u}(t)-$ a curve in $2 \mathrm{D}$ or a surface in $3 \mathrm{D}-$ is defined by a certain threshold value $c_{\mathrm{th}}$, namely,

$$
\Gamma_{u}(t)=\left\{\vec{x} \in \mathcal{B}, u(\vec{x}, t)=c_{\mathrm{th}}\right\},
$$

with the already traveled-through region given by

$$
\Omega_{u}^{\text {in }}(t)=\left\{\vec{x} \in \mathcal{B}, u(\vec{x}, t)>c_{\text {th }}\right\} .
$$


In order to characterize the location of the front, it will also be valuable to use the level set formalism [67]. We recall that for an object occupying the region $\Omega^{\text {in }} \subset \mathcal{B}$ of boundary $\Gamma$, an admissible level set is a smooth function $\phi$ such that

$$
\phi>0 \text { in } \Omega^{\text {in }}, \phi<0 \text { in } \mathcal{B} \backslash \overline{\Omega^{\text {in }}} \text { and } \phi=0 \text { on } \Gamma .
$$

We also define the single layer distribution associated with the boundary $\Gamma$, namely, the distribution $\delta_{\Gamma}$ such that

$$
\int_{\mathcal{B}} \delta_{\Gamma} \psi d \mathcal{B}=\int_{\Gamma} \psi d \Gamma
$$

for any smooth function $\psi$, where the integral in the left-hand side should be interpreted in the distribution sense. As is classical, this distribution is directly related to the usual Dirac $\delta$-function - defined in $\mathbb{R}$ - by the identity

$$
\delta_{\Gamma}=|\vec{\nabla} \phi| \delta \circ \phi,
$$

which holds for any admissible level set $\phi$ associated with $\Gamma$.

\section{Design and analysis of the observer}

Our strategy for designing an adequate state observer for the reaction-diffusion model (1) will be to take a detour via the related eikonal-curvature equation [50, 49], for which existing image processing methods pertaining to object detection in images can be used for front tracking.

\subsection{Eikonal-curvature equation and associated observer}

We start by briefly summarizing the derivation of the so-called eikonal-curvature equation related to the reaction-diffusion model (1), in order to be able to deal - in Section 3.2 below - with the modifications induced by corrections terms associated with an observer method. For more details on this derivation we refer to [50, 7, 49] and references therein.

This derivation relies on the definition of a time-dependent coordinate system $\left(\xi_{1}, \xi_{2}, \xi_{3}\right)$ designed to follow the front over time. Namely, this coordinate system is defined so that $\xi_{1}=0$ characterizes the front location, the vector

$$
\left.\frac{\partial \vec{x}}{\partial \xi_{1}}\right|_{\xi_{1}=0}
$$

is normal to the front, and $\xi_{1}$ undergoes a finite variation across the front thickness. As fronts are sharp in the types of problems considered here, this thickness of characteristic length denoted by $\varepsilon$ is small. Changing coordinates in the reaction-diffusion equation yields - assuming that the diffusion tensor is isotropic of constant diffusion coefficient $\sigma$ for the sake of simplicity in this discussion - using the Einstein implicit summation convention

$$
\partial_{t} u=\sigma a_{i l} a_{i k} \frac{\partial^{2} u}{\partial \xi_{l} \partial \xi_{k}}+\sigma \frac{\partial a_{i l}}{\partial x_{i}} \frac{\partial u}{\partial \xi_{l}}+a_{j k}\left(\partial_{t} x_{j}\right) \frac{\partial u}{\partial \xi_{k}}+k f,
$$


where $a_{i j}=\partial \xi_{j} / \partial x_{i}$, and the partial derivatives with respect to time should be understood with $\left(\xi_{1}, \xi_{2}, \xi_{3}\right)$ fixed. With the above sharpness assumption on the front we infer

$$
a_{i 1}=O\left(\varepsilon^{-1}\right),
$$

i.e. these coefficients are larger than the others by one order of $\varepsilon$. Denoting $\vec{a}=\left(a_{i 1}\right)_{i}$ and retaining only the leading order in $\varepsilon$ in the above equation we obtain

$$
\sigma|\vec{a}|^{2} \frac{\partial^{2} u}{\partial \xi_{1}^{2}}+\left(\sigma \vec{\nabla}_{\vec{x}} \cdot \vec{a}+\vec{a} \cdot \partial_{t} \vec{x}\right) \frac{\partial u}{\partial \xi_{1}}+k f(u)=0 .
$$

By a simple rescaling of the $\xi_{1}$-coordinate we can arrange so that $\sigma|\vec{a}|^{2}=k$, and using the fact that $u$ is constant on the front we are led to the ordinary differential equation

$$
u^{\prime \prime}+c_{0} u^{\prime}+f(u)=0,
$$

where the derivative is with respect to $\xi_{1}$ - the only remaining variable - and with $c_{0}$ such that

$$
k c_{0}=\sigma \vec{\nabla}_{\vec{x}} \cdot \vec{a}+\vec{a} \cdot \partial_{t} \vec{x} .
$$

It is a classical result that - under adequate assumptions on $f$ - there exists a unique constant $c_{0}$ depending on $f$ such that we can find a strictly decreasing function $u$ solution of (4), see [2]. For instance, for $f(u)=u(1-u)$, we have $c_{0}=2 \sqrt{f^{\prime}(0)}=2$. We now rewrite (5) in terms of $\phi_{u}$ a level set of the front $\Gamma_{u}$. Noting that $\vec{a}$ is normal to the front we have

$$
\vec{a}=-\sqrt{\frac{k}{\sigma}} \frac{\vec{\nabla}_{\vec{x}} \phi_{u}}{\left|\vec{\nabla}_{\vec{x}} \phi_{u}\right|} .
$$

Then, as $\phi_{u}$ is constant on the front, we have

$$
\vec{\nabla}_{\vec{x}} \phi_{u} \cdot \partial_{t} \vec{x}+\partial_{t} \phi_{u}=0 .
$$

Substituting these two identities in (5), we finally obtain the eikonal-curvature equation

$$
\partial_{t} \phi_{u}=\left|\vec{\nabla}_{\vec{x}} \phi_{u}\right|\left(\sigma \vec{\nabla}_{\vec{x}} \cdot \frac{\vec{\nabla}_{\vec{x}} \phi_{u}}{\left|\vec{\nabla}_{\vec{x}} \phi_{u}\right|}+\sqrt{\sigma k} c_{0}\right), \mathcal{B} \times(0, T) .
$$

We will now propose an observer for this level set formulation, assuming that we can use as measurements a time-continuous sequence of "images" of the traveled-through regions, namely, maps $z(t)$ taking essentially two different values inside and outside the traveled-through region, up to some perturbations and regularization across the front. We will discuss in greater detail below some adequate definitions of such maps. Denoting by $\hat{\phi}$ the observer, and by $\hat{\Gamma}$ and $\hat{\Omega}^{\text {in }}$ the associated front and traveled-through region - respectively - we propose the following dynamical equation

$$
\begin{aligned}
\partial_{t} \hat{\phi}=|\vec{\nabla} \hat{\phi}|(\sigma \vec{\nabla} & \left.\cdot\left(\frac{\vec{\nabla} \hat{\phi}}{|\vec{\nabla} \hat{\phi}|}\right)+\sqrt{\sigma k} c_{0}\right) \\
& +\lambda \delta_{\hat{\Gamma}} \alpha(|\vec{\nabla} \hat{\phi}|)\left(-\left(z-C_{1}\left(\hat{\Omega}^{\mathrm{in}}\right)\right)^{2}+\left(z-C_{2}\left(\hat{\Omega}^{\mathrm{in}}\right)\right)^{2}\right), \mathcal{B} \times(0, T),
\end{aligned}
$$


where $\lambda$ is a positive constant, $\alpha$ a strictly positive function to be prescribed, and with

$$
C_{1}\left(\hat{\Omega}^{\mathrm{in}}\right)=\frac{\int_{\hat{\Omega}^{\mathrm{in}}} z d \mathcal{B}}{\int_{\hat{\Omega}^{\mathrm{in}}} d \mathcal{B}}, \quad C_{2}\left(\hat{\Omega}^{\mathrm{in}}\right)=\frac{\int_{\mathcal{B}} \backslash \overline{\hat{\Omega}^{\mathrm{in}}} z d \mathcal{B}}{\int_{\mathcal{B}} \backslash \overline{\hat{\Omega}^{\mathrm{in}}} d \mathcal{B}} .
$$

The scalar parameter $\lambda$ is often called the gain and balances the impact of the datacorrection on the model depending on the level of confidence in the data. This justifies the nudging terminology used in the data assimilation community [53]. The gain is therefore typically adjusted by considering the level of noise in the data, but also by evaluating the increased stability introduced by the data correction. Sometimes an excessively large gain can be counter-productive - see a striking example in [63] - and in any case should eventually be optimized - see [86, 79, 91].

Note that (7) was obtained by incorporating a correction in the eikonal-curvature equation (6). This correction term uses the information contained in the data, and this specific expression is inspired from the method of active contours without edges [67, 17], see also below for an interpretation based on the gradient projection method.

\subsection{Front-based observer for reaction-diffusion model}

As there is more relevant information in the solution of the reaction-diffusion problem than in the corresponding level set - i.e. we are interested in the actual distributed values of the physical quantity $u$ over time - we aim at defining an observer directly for the original problem. Nevertheless, as we are confident in the ability of the above-defined observer to track the front, we will seek a consistent observer definition, namely, such that (7) is obtained as the limit in the asymptotic analysis outlined in our discussion of Section 3.1. By inspection, we propose the following observer formulation.

$$
\begin{aligned}
\partial_{t} \hat{u} & =\vec{\nabla} \cdot(\overrightarrow{\vec{\sigma}} \cdot \vec{\nabla} \hat{u})+k f(\hat{u}) \\
& +\lambda \delta_{\Gamma_{\hat{u}}} \frac{\alpha\left(\left|\vec{\nabla} \phi_{\hat{u}}\right|\right)}{\left|\vec{\nabla} \phi_{\hat{u}}\right|} \frac{\vec{\nabla} \phi_{\hat{u}}}{\left|\vec{\nabla} \phi_{\hat{u}}\right|} \cdot \vec{\nabla} \hat{u}\left(-\left(z-C_{1}\left(\Omega_{\hat{u}}^{\text {in }}\right)\right)^{2}+\left(z-C_{2}\left(\Omega_{\hat{u}}^{\text {in }}\right)\right)^{2}\right), \mathcal{B} \times(0, T),
\end{aligned}
$$

where now $\Gamma_{\hat{u}}$ denotes the front associated with $\hat{u}, \Omega_{\hat{u}}^{\text {in }}$ the corresponding traveled-through region, and $\phi_{\hat{u}}$ a level set consistent with $\hat{u}$. Using the above asymptotic analysis technique, the following result is readily established.

Proposition 1. Assuming that the diffusion tensor is isotropic with constant coefficient $\sigma,(7)$ is the modified eikonal-curvature equation associated with (9).

PROOF. Performing the same change of coordinates as in the above formal asymptotic analysis, Eq.(3) expressing the dominant term in the expansion is modified into

$$
\begin{array}{r}
\sigma|\vec{a}|^{2} \frac{\partial^{2} \hat{u}}{\partial \xi_{1}^{2}}+\left[\sigma \vec{\nabla}_{\vec{x}} \cdot \vec{a}+\vec{a} \cdot \partial_{t} \vec{x}+\lambda|\vec{a}| \delta_{\Gamma_{\hat{u}}} \frac{\alpha\left(\left|\vec{\nabla} \phi_{\hat{u}}\right|\right)}{\left|\vec{\nabla} \phi_{\hat{u}}\right|}\left(\left(z-C_{1}\left(\Omega_{\hat{u}}^{\text {in }}\right)\right)^{2}-\left(z-C_{2}\left(\Omega_{\hat{u}}^{\text {in }}\right)\right)^{2}\right)\right] \frac{\partial \hat{u}}{\partial \xi_{1}} \\
+k f(\hat{u})=0, \quad(10)
\end{array}
$$

where we have used the identity

$$
\frac{\vec{\nabla} \phi_{\hat{u}}}{\left|\vec{\nabla} \phi_{\hat{u}}\right|} \cdot \vec{\nabla} \hat{u}=-|\vec{a}| \frac{\partial \hat{u}}{\partial \xi_{1}}
$$


Then (5) is transformed into

$$
k c_{0}=\sigma \vec{\nabla}_{\vec{x}} \cdot \vec{a}+\vec{a} \cdot \partial_{t} \vec{x}+\lambda|\vec{a}| \delta_{\Gamma_{\hat{u}}} \frac{\alpha\left(\left|\vec{\nabla} \phi_{\hat{u}}\right|\right)}{\left|\vec{\nabla} \phi_{\hat{u}}\right|}\left(\left(z-C_{1}\left(\Omega_{\hat{u}}^{\text {in }}\right)\right)^{2}-\left(z-C_{2}\left(\Omega_{\hat{u}}^{\text {in }}\right)\right)^{2}\right),
$$

and finally the same manipulation as before directly shows that the level set $\phi_{\hat{u}}$ satisfies exactly Eq.(7).

Note that we can take $\hat{u}-c_{\text {th }}$ as a particular choice of admissible level set in (9). In this case, the proposed observer equation simplifies into

$$
\begin{aligned}
\partial_{t} \hat{u}=\vec{\nabla} \cdot(\overrightarrow{\vec{\sigma}} \cdot \vec{\nabla} \hat{u})+k f(\hat{u}) & \\
& +\lambda \delta_{\Gamma_{\hat{u}}} \alpha(|\vec{\nabla} \hat{u}|)\left(-\left(z-C_{1}\left(\Omega_{\hat{u}}^{\text {in }}\right)\right)^{2}+\left(z-C_{2}\left(\Omega_{\hat{u}}^{\text {in }}\right)\right)^{2}\right), \mathcal{B} \times(0, T) .
\end{aligned}
$$

This is the form that we will use in the rest of the paper.

\subsection{Analysis of the observer}

In order to analyse the convergence of the observer we will study the estimation error defined by $\tilde{u}=u-\hat{u}$. Subtracting (12) from the state equation in (1), and taking into account the boundary and initial conditions, we obtain

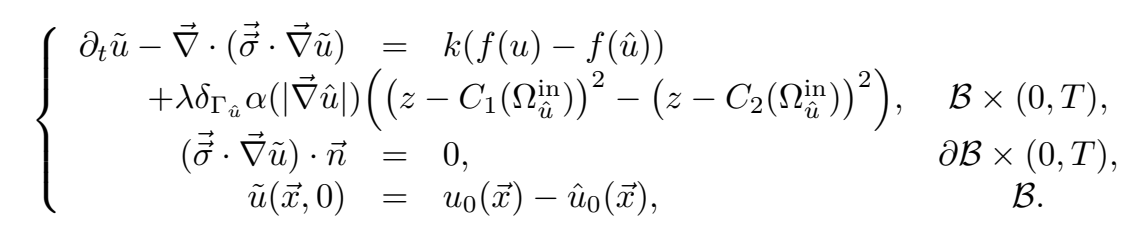

This problem can be rewritten in variational form, by which $\tilde{u}$ is the solution of

$$
\begin{aligned}
\int_{\mathcal{B}} \partial_{t} \tilde{u} v d \mathcal{B}+ & \int_{\mathcal{B}}(\overrightarrow{\vec{\sigma}} \cdot \vec{\nabla} \tilde{u}) \cdot \vec{\nabla} v d \mathcal{B}=\int_{\mathcal{B}} k(f(u)-f(\hat{u})) v d \mathcal{B} \\
& +\lambda \int_{\Gamma_{\hat{u}}} \alpha(|\vec{\nabla} \hat{u}|)\left(\left(z-C_{1}\left(\Omega_{\hat{u}}^{\text {in }}\right)\right)^{2}-\left(z-C_{2}\left(\Omega_{\hat{u}}^{\text {in }}\right)\right)^{2}\right) v d \Gamma
\end{aligned}
$$

at any time $t$ and for any suitable test function $v$, while $u$ and $\hat{u}$ respectively satisfy

$$
\int_{\mathcal{B}} \partial_{t} u v d \mathcal{B}+\int_{\mathcal{B}}(\overrightarrow{\vec{\sigma}} \cdot \vec{\nabla} u) \cdot \vec{\nabla} v d \mathcal{B}=\int_{\mathcal{B}} k f(u) v d \mathcal{B},
$$

and

$$
\begin{aligned}
\int_{\mathcal{B}} \partial_{t} \hat{u} v d \mathcal{B}+ & \int_{\mathcal{B}}(\vec{\sigma} \cdot \vec{\nabla} \hat{u}) \cdot \vec{\nabla} v d \mathcal{B}=\int_{\mathcal{B}} k f(\hat{u}) v d \mathcal{B} \\
& +\lambda \int_{\Gamma_{\hat{u}}} \alpha(|\vec{\nabla} \hat{u}|)\left(-\left(z-C_{1}\left(\Omega_{\hat{u}}^{\text {in }}\right)\right)^{2}+\left(z-C_{2}\left(\Omega_{\hat{u}}^{\text {in }}\right)\right)^{2}\right) v d \Gamma .
\end{aligned}
$$

First, in order to have a consistent observer, we expect the correction term to vanish when $\hat{u}=u$, namely, we should have on the exact front $\Gamma_{u}$

$$
\left(z-C_{1}\left(\Omega_{u}^{\text {in }}\right)\right)^{2}-\left(z-C_{2}\left(\Omega_{u}^{\text {in }}\right)\right)^{2}=0 .
$$

We are thus led to the following assumption. 
Assumption 1. On the front $\Gamma_{u}$, the data satisfy $z=\frac{C_{1}\left(\Omega_{u}^{i n}\right)+C_{2}\left(\Omega_{u}^{i n}\right)}{2}$.

Note that $C_{1}\left(\Omega_{u}^{\text {in }}\right)$ is the average of $z$ inside the traveled-through region $\Omega_{u}^{\text {in }}$, while $C_{2}\left(\Omega_{u}^{\text {in }}\right)$ is the average outside of this region. Of course, in practice it may be difficult to satisfy exactly this assumption, which means that some consistency errors - expected to be small - will be present in the system. We do not consider these errors here.

A complete mathematical analysis of the observer error in (13) is out of the scope of the present paper, due to the complexity induced by both the nonlinear reaction term and the observer correction. Our objective here will be to consider the linearized version of this equation - relevant for "sufficiently small" estimation errors around the reference trajectory - and assess the stabilization effect of the correction term. Let us define the linear form associated with the correction term

$$
\mathcal{Q}_{\hat{u}}(v)=\int_{\Gamma_{\hat{u}}} \alpha(|\vec{\nabla} \hat{u}|)\left(\left(z-C_{1}\left(\Omega_{\hat{u}}^{\mathrm{in}}\right)\right)^{2}-\left(z-C_{2}\left(\Omega_{\hat{u}}^{\mathrm{in}}\right)\right)^{2}\right) v d \Gamma .
$$

The first step will then consist in differentiating this term with respect to $\hat{u}$, which amounts to finding the derivative of the functional

$$
\tau \mapsto \mathcal{Q}_{\hat{u}+\tau \psi}(v),
$$

in $\tau=0$, for any given - sufficiently regular - scalar field $\psi$. As $\hat{u}$ mostly enters in $\mathcal{Q}_{\hat{u}}(v)$ via the shape of the contour $\Gamma_{\hat{u}}$, we will use the theory of shape derivatives, see e.g. $[34,43]$ and references therein. A simple geometric reasoning shows that the contour velocity associated with $\hat{u}+\tau \psi=c_{\text {th }}$ at $\tau=0$ - when seeing $\tau$ as a "pseudo-time" variable - is given by

$$
\vec{\psi}=\frac{\psi}{|\vec{\nabla} \hat{u}|} \vec{n}_{\hat{u}}
$$

where $\vec{n}_{\hat{u}}=-\vec{\nabla} \hat{u} /|\vec{\nabla} \hat{u}|$ is a unit vector normal to $\Gamma_{\hat{u}}$. We will prove the following result.

Proposition 2. Assuming that the contour $\Gamma_{\hat{u}}$ is closed, the Fréchet derivative of $\mathcal{Q}_{\hat{u}}(v)$ in the direction $\psi$ is given by

$$
\begin{aligned}
\left\langle d_{\hat{u}} \mathcal{Q}_{\hat{u}}(v)\right. & , \psi\rangle=\int_{\Gamma_{\hat{u}}} \kappa \alpha(|\vec{\nabla} \hat{u}|)\left(\left(z-C_{1}\left(\Omega_{\hat{u}}^{i n}\right)\right)^{2}-\left(z-C_{2}\left(\Omega_{\hat{u}}^{i n}\right)\right)^{2}\right) v \frac{\psi}{|\vec{\nabla} \hat{u}|} d \Gamma \\
& -\int_{\Gamma_{\hat{u}}} \alpha^{\prime}(|\vec{\nabla} \hat{u}|)\left(\left(z-C_{1}\left(\Omega_{\hat{u}}^{i n}\right)\right)^{2}-\left(z-C_{2}\left(\Omega_{\hat{u}}^{i n}\right)\right)^{2}\right) v \partial_{n} \psi d \Gamma \\
& -\int_{\Gamma_{\hat{u}}} \alpha^{\prime}(|\vec{\nabla} \hat{u}|) \partial_{n}^{2} \hat{u}\left(\left(z-C_{1}\left(\Omega_{\hat{u}}^{i n}\right)\right)^{2}-\left(z-C_{2}\left(\Omega_{\hat{u}}^{i n}\right)\right)^{2}\right) v \frac{\psi}{|\vec{\nabla} \hat{u}|} d \Gamma \\
& +2 \int_{\Gamma_{\hat{u}}} \alpha(|\vec{\nabla} \hat{u}|) \partial_{n} z\left(C_{2}\left(\Omega_{\hat{u}}^{i n}\right)-C_{1}\left(\Omega_{\hat{u}}^{i n}\right)\right) v \frac{\psi}{|\vec{\nabla} \hat{u}|} d \Gamma \\
& -2 \frac{1}{\left|\Omega_{\hat{u}}^{i n}\right|} \int_{\Gamma_{\hat{u}}}\left(z-C_{1}\left(\Omega_{\hat{u}}^{i n}\right)\right) \frac{\psi}{|\vec{\nabla} \hat{u}|} d \Gamma \times \int_{\Gamma_{\hat{u}}} \alpha(|\vec{\nabla} \hat{u}|)\left(z-C_{1}\left(\Omega_{\hat{u}}^{i n}\right)\right) v d \Gamma \\
& -2 \frac{1}{\left|\mathcal{B} \backslash \overrightarrow{\Omega_{\hat{u}}^{i n}}\right|} \int_{\Gamma_{\hat{u}}}\left(z-C_{2}\left(\Omega_{\hat{u}}^{i n}\right)\right) \frac{\psi}{|\vec{\nabla} \hat{u}|} d \Gamma \times \int_{\Gamma_{\hat{u}}} \alpha(|\vec{\nabla} \hat{u}|)\left(z-C_{2}\left(\Omega_{\hat{u}}^{i n}\right)\right) v d \Gamma \\
& +\int_{\Gamma_{\hat{u}}} \alpha(|\vec{\nabla} \hat{u}|)\left(\left(z-C_{1}\left(\Omega_{\hat{u}}^{i n}\right)\right)^{2}-\left(z-C_{2}\left(\Omega_{\hat{u}}^{i n}\right)\right)^{2}\right) \partial_{n} v \frac{\psi}{|\vec{\nabla} \hat{u}|} d \Gamma
\end{aligned}
$$


where $\kappa$ denotes the so-called additive curvature of the contour $\Gamma_{\hat{u}}$, and $\partial_{n}$ the spatial derivative in the direction normal to the contour.

ProOF. Differentiating $\mathcal{Q}_{\hat{u}+\tau \psi}(v)$ with respect to $\tau$ amounts to finding the derivative of a functional of the type

$$
\int_{\Gamma_{\tau}} \mathcal{F}\left(\tau, \Gamma_{\tau}, \vec{x}\right) d \Gamma
$$

where the direct dependence in $\tau$ of the functional $\mathcal{F}$ is due to the term $\alpha(|\vec{\nabla}(\hat{u}+\tau \psi)|)$. Knowing the flow $\vec{\psi}$ given by (16) that displaces the contour, this can be seen as a shape derivative problem. We use the shape derivative formula (.1) proven in Proposition 4 below (Appendix), as a quite straightforward extension of [43, Prop. 2]. Since the flow $\vec{\psi}$ is normal to the contour - recall $(16)$ - this gives here

$$
\left\langle d_{\hat{u}} \mathcal{Q}_{\hat{u}}(v), \psi\right\rangle=\int_{\Gamma_{\hat{u}}}\left(\dot{\mathcal{F}}+\kappa \mathcal{F} \frac{\psi}{|\vec{\nabla} \hat{u}|}\right) d \Gamma,
$$

with $\dot{\mathcal{F}}$ the particle derivative given by

$$
\dot{\mathcal{F}}=\left.\partial_{\tau} \mathcal{F}\left(\tau, \Gamma_{\tau}, \vec{x}+\tau \vec{\psi}\right)\right|_{\tau=0}
$$

Considering first the particle derivative of $\alpha(|\vec{\nabla}(\hat{u}+\tau \psi)|)$ we have

$$
\begin{aligned}
\dot{\alpha} & =\partial_{\tau} \alpha(|\vec{\nabla}(\hat{u}+\tau \psi)|)+\vec{\nabla}(\alpha(|\vec{\nabla} \hat{u}|)) \cdot \vec{\psi} \\
& =\alpha^{\prime}(|\vec{\nabla} \hat{u}|)\left(\frac{\vec{\nabla} \hat{u}}{|\vec{\nabla} \hat{u}|} \cdot \vec{\nabla} \psi+\vec{\nabla}|\vec{\nabla} \hat{u}| \cdot \vec{\psi}\right) \\
& =-\alpha^{\prime}(|\vec{\nabla} \hat{u}|)\left(\partial_{n} \psi+\frac{\psi}{|\vec{\nabla} \hat{u}|} \partial_{n}^{2} \hat{u}\right),
\end{aligned}
$$

where we have used the facts that $\vec{\nabla} \hat{u} /|\vec{\nabla} \hat{u}|=-\vec{n}_{\hat{u}}$ and $|\vec{\nabla} \hat{u}|=-\partial_{n} \hat{u}$. Next, as $C_{1}\left(\Omega_{\hat{u}}^{\text {in }}\right)$ and $C_{2}\left(\Omega_{\hat{u}}^{\text {in }}\right)$ are scalars that only depend on the shape - recall $(8)$ - the particle and shape derivatives are the same, and

$$
\begin{aligned}
\dot{C}_{1} & =\frac{\int_{\Gamma_{\hat{u}}} z \vec{\psi} \cdot \vec{n}_{\hat{u}} d \Gamma \times \int_{\Omega_{\hat{u}}^{\text {in }}} d \mathcal{B}-\int_{\Gamma_{\hat{u}}} \vec{\psi} \cdot \vec{n}_{\hat{u}} d \Gamma \times \int_{\Omega_{\hat{u}}^{\text {in }}} z d \mathcal{B}}{\left(\int_{\Omega_{\hat{u}}^{\text {in }}} d \mathcal{B}\right)^{2}} \\
& =\frac{1}{\left|\Omega_{\hat{u}}^{\text {in }}\right|} \int_{\Gamma_{\hat{u}}}\left(z-C_{1}\left(\Omega_{\hat{u}}^{\text {in }}\right)\right) \vec{\psi} \cdot \vec{n}_{\hat{u}} d \Gamma,
\end{aligned}
$$

while likewise

$$
\dot{C}_{2}=-\frac{1}{\left|\mathcal{B} \backslash \overline{\Omega_{\hat{u}}^{\text {in }}}\right|} \int_{\Gamma_{\hat{u}}}\left(z-C_{2}\left(\Omega_{\hat{u}}^{\text {in }}\right)\right) \vec{\psi} \cdot \vec{n}_{\hat{u}} d \Gamma .
$$

Finally, $z$ and $v$ only have spatial derivatives, to be taken in the direction of $\vec{\psi}$ as given in (16). Gathering all the terms, we obtain the expression (17). 
Based on (13), the variational formulation satisfied by the linearized error - also denoted by $\tilde{u}$ for simplicity - reads

$$
\int_{\mathcal{B}} \partial_{t} \tilde{u} v d \mathcal{B}+\int_{\mathcal{B}}(\overrightarrow{\vec{\sigma}} \cdot \vec{\nabla} \tilde{u}) \cdot \vec{\nabla} v d \mathcal{B}=-\int_{\mathcal{B}} k\left(d_{u} f(u) ; \tilde{u}\right) v d \mathcal{B}-\left\langle d_{\hat{u}} \mathcal{Q}_{\hat{u}}(v), \tilde{u}\right\rangle,
$$

where many terms in (17) vanish due to Assumption 1, since we are taking the differential around $\hat{u}=u$, namely, we have here

$$
\begin{aligned}
& \left\langle d_{\hat{u}} \mathcal{Q}_{\hat{u}}(v), \tilde{u}\right\rangle=2\left(C_{2}\left(\Omega_{u}^{\mathrm{in}}\right)-C_{1}\left(\Omega_{u}^{\mathrm{in}}\right)\right) \int_{\Gamma_{u}} \alpha(|\vec{\nabla} u|) \partial_{n} z \frac{\tilde{u}}{|\vec{\nabla} u|} v d \Gamma \\
& -2\left(\frac{C_{2}\left(\Omega_{u}^{\mathrm{in}}\right)-C_{1}\left(\Omega_{u}^{\mathrm{in}}\right)}{2}\right)^{2}\left(\frac{1}{\left|\Omega_{u}^{\mathrm{in}}\right|}+\frac{1}{\left|\mathcal{B} \backslash \overline{\Omega_{u}^{\text {in }}}\right|}\right) \int_{\Gamma_{u}} \frac{\tilde{u}}{|\vec{\nabla} u|} d \Gamma \times \int_{\Gamma_{u}} \alpha(|\vec{\nabla} u|) v d \Gamma .
\end{aligned}
$$

Note that (19) is a linear homogeneous equation in $\tilde{u}$, as the observer will start with an error in the initial condition, indeed, and we expect this error to be driven to zero as soon as possible. In fact, when taking $v=\tilde{u}$ in (19) we obtain the energy estimate

$$
\frac{d}{d t}\left(\frac{1}{2}\|\tilde{u}\|_{L^{2}(\mathcal{B})}^{2}\right)=-\int_{\mathcal{B}}(\overrightarrow{\vec{\sigma}} \cdot \vec{\nabla} \tilde{u}) \cdot \vec{\nabla} \tilde{u} d \mathcal{B}-\int_{\mathcal{B}} k\left(d_{u} f(u) ; \tilde{u}\right) \tilde{u} d \mathcal{B}-\left\langle d_{\hat{u}} \mathcal{Q}_{\hat{u}}(\tilde{u}), \tilde{u}\right\rangle .
$$

As the diffusion tensor is always positive, the corresponding term in the right-hand side is energy-decreasing as usual. The effect of the reaction term is problem-dependent, and is outside of our scope here. As regards the observer correction term, we obtain a beneficial energy-decreasing effect if

$$
\left\langle d_{\hat{u}} \mathcal{Q}_{\hat{u}}(\tilde{u}), \tilde{u}\right\rangle \geq 0 .
$$

This condition is not automatically satisfied, as e.g. when choosing $\alpha(|\vec{\nabla} u|)=1 /|\vec{\nabla} u|$ the second term in (20) brings a negative contribution. This simply means that we need some additional (reasonable) assumption on the data $z$. Let us define the characteristic length $h_{z_{u}}$ by

$$
\frac{1}{h_{z_{u}}}=\min _{\vec{x} \in \Gamma_{u}} \frac{\left|\partial_{n} z\right|}{\left|C_{1}\left(\Omega_{u}^{\text {in }}\right)-C_{2}\left(\Omega_{u}^{\text {in }}\right)\right|},
$$

and we can interpret $h_{z_{u}}$ as the wavelength of the front width in the data. We can now show the following result concerning the observer effect.

Proposition 3. Assuming that we have

$$
\frac{1}{h_{z_{u}}} \geq \frac{1}{4}\left(\frac{1}{\left|\Omega_{u}^{i n}\right|}+\frac{1}{\left|\mathcal{B} \backslash \overline{\Omega_{u}^{i n}}\right|}\right)\left(\int_{\Gamma_{u}} \alpha(|\vec{\nabla} u|)|\vec{\nabla} u| d \Gamma \times \int_{\Gamma_{u}} \frac{1}{\alpha(|\vec{\nabla} u|)|\vec{\nabla} u|} d \Gamma\right)^{\frac{1}{2}},
$$

then the condition (21) is satisfied, hence the observer effect is energy-decreasing in the linearized error. 
Proof. By the Cauchy-Schwarz inequality we have

$$
\begin{aligned}
& \left|\int_{\Gamma_{u}} \frac{1}{|\vec{\nabla} u|} \tilde{u} d \Gamma_{u} \int_{\Gamma_{u}} \alpha(|\vec{\nabla} u|) \tilde{u} d \Gamma\right| \\
& \quad=\left|\int_{\Gamma_{u}} \sqrt{\frac{\alpha(|\vec{\nabla} u|)}{|\vec{\nabla} u|}} \tilde{u} \frac{1}{\sqrt{\alpha(|\vec{\nabla} u|)|\vec{\nabla} u|}} d \Gamma \times \int_{\Gamma_{u}} \sqrt{\frac{\alpha(|\vec{\nabla} u|)}{|\vec{\nabla} u|}} \tilde{u} \sqrt{\alpha(|\vec{\nabla} u|)|\vec{\nabla} u|} d \Gamma\right| \\
& \leq \int_{\Gamma_{u}} \frac{\alpha(|\vec{\nabla} u|)}{|\vec{\nabla} u|} \tilde{u}^{2} d \Gamma\left(\int_{\Gamma_{u}} \alpha(|\vec{\nabla} u|)|\vec{\nabla} u| d \Gamma \times \int_{\Gamma_{u}} \frac{1}{\alpha(|\vec{\nabla} u|)|\vec{\nabla} u|} d \Gamma\right)^{\frac{1}{2}} .
\end{aligned}
$$

Recalling (20) we then infer

$$
\begin{aligned}
& \left\langle d_{\hat{u}} \mathcal{Q}_{\hat{u}}(\tilde{u}), \tilde{u}\right\rangle \\
& \geq 2\left(C_{2}\left(\Omega_{u}^{\mathrm{in}}\right)-C_{1}\left(\Omega_{u}^{\mathrm{in}}\right)^{2} \frac{1}{h_{z_{u}}} \int_{\Gamma_{u}} \frac{\alpha(|\vec{\nabla} u|)}{|\vec{\nabla} u|} \tilde{u}^{2} d \Gamma\right. \\
& \quad-2\left(\frac{C_{2}\left(\Omega_{u}^{\mathrm{in}}\right)-C_{1}\left(\Omega_{u}^{\mathrm{in}}\right.}{2}\right)^{2}\left(\frac{1}{\left|\Omega_{\hat{u}}^{\mathrm{in}}\right|}+\frac{1}{\mid \mathcal{B} \backslash \overline{\Omega_{\hat{u}}^{\mathrm{in}} \mid}}\right) \\
& \times\left(\int_{\Gamma_{u}} \alpha(|\vec{\nabla} u|)|\vec{\nabla} u| d \Gamma \times \int_{\Gamma_{u}} \frac{1}{\alpha(|\vec{\nabla} u|)|\vec{\nabla} u|} d \Gamma\right)^{\frac{1}{2}} \int_{\Gamma_{u}} \frac{\alpha(|\vec{\nabla} u|)}{|\vec{\nabla} u|} \tilde{u}^{2} d \Gamma \\
& \geq\left(\frac{1}{h_{z_{u}}}-\frac{1}{4}\left(\frac{1}{\left|\Omega_{\hat{u}}^{\mathrm{in}}\right|}+\frac{1}{\mid \mathcal{B} \backslash \overline{\Omega_{\hat{u}}^{\mathrm{in}} \mid}}\right)\left(\int_{\Gamma_{u}} \alpha(|\vec{\nabla} u|)|\vec{\nabla} u| d \Gamma \times \int_{\Gamma_{u}} \frac{1}{\alpha(|\vec{\nabla} u|)|\vec{\nabla} u|} d \Gamma\right)^{\frac{1}{2}}\right) \\
& \times 2\left(C_{2}\left(\Omega_{u}^{\mathrm{in}}\right)-C_{1}\left(\Omega_{u}^{\mathrm{in}}\right)^{2} \int_{\Gamma_{u}} \frac{\alpha(|\vec{\nabla} u|)}{|\vec{\nabla} u|} \tilde{u}^{2} d \Gamma,\right.
\end{aligned}
$$

which by (22) entails the desired positivity.

Condition (22) pertains to the sharpness of the front in the data - in other words to image contrast - for which conditions are also required in image processing, see e.g. [43]. We can further analyse this condition by noting that

$$
\left(\int_{\Gamma_{u}} \alpha(|\vec{\nabla} u|)|\vec{\nabla} u| d \Gamma \times \int_{\Gamma_{u}} \frac{1}{\alpha(|\vec{\nabla} u|)|\vec{\nabla} u|} d \Gamma\right)^{\frac{1}{2}} \geq \int_{\Gamma_{u}} \frac{\alpha(|\vec{\nabla} u|)|\vec{\nabla} u|}{\alpha(|\vec{\nabla} u|)|\vec{\nabla} u|} d \Gamma=\left|\Gamma_{u}\right|,
$$

by the Cauchy-Schwarz inequality, and that the inequality is an equality when

$$
\alpha(|\vec{\nabla} u|)=\frac{1}{|\vec{\nabla} u|} .
$$

Therefore, this particular choice of $\alpha$ function is that for which the condition (22) is the least restrictive for the data. We will adhere to this choice in the sequel, in which case the condition takes the simpler form

$$
\frac{1}{h_{z_{u}}} \geq \frac{1}{4}\left(\frac{1}{\left|\Omega_{u}^{\text {in }}\right|}+\frac{1}{\left|\mathcal{B} \backslash \overline{\Omega_{u}^{\text {in }}}\right|}\right)\left|\Gamma_{u}\right|
$$


and by considering specific examples of shapes one can readily see that this condition is very easily satisfied.

Finally, we will provide an interpretation of our observer strategy by establishing a connection with a gradient projection method. Let us consider the functional

$$
\mathcal{J}_{\hat{u}}=\int_{\Omega_{\hat{u}}^{\text {in }}}\left(z-C_{1}\left(\Omega_{\hat{u}}^{\text {in }}\right)\right)^{2} d \mathcal{B}+\int_{\mathcal{B} \backslash \overline{\Omega_{\hat{u}}^{\text {in }}}}\left(z-C_{2}\left(\Omega_{\hat{u}}^{\text {in }}\right)\right)^{2} d \mathcal{B} .
$$

Clearly, when the data are perfectly contrasted - namely, $z$ takes two different values $C_{1}\left(\Omega_{u}^{\text {in }}\right)$ and $C_{2}\left(\Omega_{u}^{\text {in }}\right)$ inside and outside of $\Omega_{u}^{\text {in }}$, respectively - this functional provides a good criterion for comparing the fronts of $u$ and $\hat{u}$, as the functional can only vanish when the contours $\Gamma_{u}$ and $\Gamma_{\hat{u}}$ perfectly coincide (assuming adequate regularity). More generally - i.e. without the perfect contrast assumption - we will compute the derivative of the criterion. Using shape derivative theory as in the proof of Proposition 2 - albeit here in a simpler case - we find

$$
\begin{aligned}
\left\langle d_{\hat{u}} \mathcal{J}_{\hat{u}}, \psi\right\rangle= & -2 \dot{C}_{1} \int_{\Omega_{\hat{u}}^{\text {in }}}\left(z-C_{1}\left(\Omega_{\hat{u}}^{\text {in }}\right)\right) d \mathcal{B}-2 \dot{C}_{2} \int_{\mathcal{B} \backslash \overline{\Omega_{\hat{u}}^{\text {in }}}}\left(z-C_{2}\left(\Omega_{\hat{u}}^{\text {in }}\right)\right) d \mathcal{B} \\
& +\int_{\Gamma_{\hat{u}}}\left(\left(z-C_{1}\left(\Omega_{\hat{u}}^{\text {in }}\right)\right)^{2}-\left(z-C_{2}\left(\Omega_{\hat{u}}^{\text {in }}\right)\right)^{2}\right) \vec{\psi} \cdot \vec{n}_{\hat{u}} d \Gamma \\
= & \int_{\Gamma_{\hat{u}}}\left(\left(z-C_{1}\left(\Omega_{\hat{u}}^{\text {in }}\right)\right)^{2}-\left(z-C_{2}\left(\Omega_{\hat{u}}^{\text {in }}\right)\right)^{2}\right) \frac{\psi}{|\vec{\nabla} \hat{u}|} d \Gamma,
\end{aligned}
$$

since we have by definition of $C_{1}$ and $C_{2}$

$$
\int_{\Omega_{\hat{u}}^{\text {in }}}\left(z-C_{1}\left(\Omega_{\hat{u}}^{\text {in }}\right)\right) d \mathcal{B}=\int_{\mathcal{B} \backslash \overline{\Omega_{\hat{u}}^{\text {in }}}}\left(z-C_{2}\left(\Omega_{\hat{u}}^{\text {in }}\right)\right) d \mathcal{B}=0 .
$$

Therefore, we can formally define the gradient of $\mathcal{J}_{\hat{u}}$ as

$$
\nabla \mathcal{J}_{\hat{u}}=\frac{\delta_{\Gamma_{\hat{u}}}}{|\vec{\nabla} \hat{u}|}\left(\left(z-C_{1}\left(\Omega_{\hat{u}}^{\mathrm{in}}\right)\right)^{2}-\left(z-C_{2}\left(\Omega_{\hat{u}}^{\mathrm{in}}\right)\right)^{2}\right),
$$

and we can see that the observer correction term in (12) is nothing but $-\lambda \nabla \mathcal{J}_{\hat{u}}$ with our particular choice of $\alpha$ function given in (23), namely, this is a gradient descent term quite similar to gradient projection methods in image processing [90]. We can further point out that Assumption 1 ensures that the gradient vanishes when $\Gamma_{u}$ and $\Gamma_{\hat{u}}$ coincide. Moreover, the positivity property (21) in fact also represents the positivity of the Hessian of $\mathcal{J}_{\hat{u}}$, which guarantees that $u$ provides a local minimum - since the positivity is in fact strict provided the inequality in $(22)$ is also strict, as can be checked in the proof of Proposition 3.

\subsection{Numerical discretization}

We start here with the spatial discretization of the model and observer, for which finite difference or finite element can be considered. Assuming a finite difference scheme - well-adapted to $1 \mathrm{D}$ problems where $\mathcal{B}$ is an interval, or to higher dimensions where the domain is a simple box - the discretization is directly performed with the strong formulation. The main difficulty then lies in adequately discretizing the $\delta_{\hat{\Gamma}}$ function in 
the observer equation (12). This question is well-addressed in the literature for immersed boundary problems [10] or for level-set formulations [78, 67]. Here, we rely on [35] and the relation (2) linking the single layer distribution to a standard Dirac function. We introduce the real-valued continuous functions $\psi(x)=\frac{1}{2}(1+\cos (\pi x))$, and a discretization of the Dirac function in 1D given by

$$
\delta_{\rho}(x)=\left\{\begin{array}{cl}
\frac{1}{\rho(x)} \psi\left(\frac{x}{\rho(x)}\right) & \text { if }|x| \leq \rho(x), \\
0 & \text { if }|x|>\rho(x)
\end{array}\right.
$$

with $\rho$ a strictly positive function. In our case, we need to discretize (2) for the level set $\phi=\hat{u}-c_{\mathrm{th}}$. Following the discretization advocated in [35], the function $\rho$ is defined by

$$
\rho=\frac{|\vec{\nabla} \phi|_{1}}{|\vec{\nabla} \phi|} \rho_{0}
$$

where $|\cdot|_{1}$ denotes the 1 -norm - by contrast to the Euclidean norm $|\cdot|$ - and $\rho_{0}$ a strictly positive constant. Then the following approximation is employed as in [35]

$$
\delta_{\Gamma_{\hat{u}}}=|\vec{\nabla} \phi| \delta \circ \phi=\delta\left(\frac{\phi}{|\vec{\nabla} \phi|}\right) \simeq \delta_{\rho}\left(\frac{\phi}{|\vec{\nabla} \phi|}\right)=|\vec{\nabla} \phi| \delta_{\rho_{0}|\vec{\nabla} \phi|_{1}}(\phi),
$$

to be compared to $(2)$.

As for finite element discretizations, we rely on the same type of discretization strategy for the singular front term, see [37] and references therein. Note that, in general, very special care may be required concerning the finite element basis function choice and integration rules. We should also mention that methods based on adaptive meshing can be employed to obtain a global mesh containing a conforming sub-mesh of $\Gamma_{\hat{u}}$ over time $[40,33]$, thus allowing to easily compute surface integrals in the weak formulation of the observer.

Concerning the time discretization, the specificity of reaction-diffusion systems allows to rely on splitting time schemes [3]. Typically, let $N_{T} \in \mathbb{N}^{*}$ be a given integer and consider a uniform partition $\left\{t_{n}, t_{n+1}\right\}_{0 \leq n \leq N_{T}-1}$ with $t_{n}=n T / N_{T}=n \Delta t$ of the time interval $[0, T]$. We introduce $\hat{U}=\left(\hat{U}_{n}\right)_{n}$ the approximation of $(\hat{u}(\cdot, n \Delta t))_{n}$. Then, for all $n$ we introduce intermediate steps $\left(\hat{U}_{n, k}\right)_{0 \leq k \leq N_{\text {split }}}$ such that $\hat{U}_{n, 0}=\hat{U}_{n}$ and $\hat{U}_{n, N_{\text {split }}}=\hat{U}_{n+1}$. For the sake of simplicity, let us consider $\overrightarrow{\vec{\sigma}}$ constant in space and time, and parametrized by $\overrightarrow{\vec{\sigma}}=\theta \overrightarrow{\vec{\sigma}}_{0}$. Denoting by $K$ the matrix associated with the diffusion operator with tensor $\overrightarrow{\vec{\sigma}}_{0}$, we define $K^{\theta}$ by $K^{\theta}=\theta K$, and we denote by $\mathrm{F}(\cdot)$ the space discretization of $k f(\cdot)$. Then the first two steps are

$$
\mid \begin{aligned}
& M \frac{\hat{U}_{n, 1}-\hat{U}_{n, 0}}{\Delta t}=F\left(\hat{U}_{n, 0}\right) \\
& M \frac{\hat{U}_{n, 2}-\hat{U}_{n, 1}}{\Delta t}=K^{\theta} \hat{U}_{n, 2}
\end{aligned}
$$

where $M$ denotes either the identity matrix in a finite difference scheme based on a strong formulation, or the matrix associated with the $L^{2}$ dot-product in a finite element scheme based on a variational formulation. The next step consists in introducing the 
observer correction. We define $Z_{n}$ as the discrete observation, $\hat{\Phi}_{n}$ as the discrete level set associated with $\hat{U}_{n, 2}$, and $H\left(\hat{\Phi}_{n}\right)$ as the Heaviside function applied to each pointwise value of $\hat{\Phi}_{n}$. The mean values expressed in (8) can be approximated by

$$
C_{1}\left(\hat{\Phi}_{n}, Z_{n}\right)=\frac{H\left(\hat{\Phi}_{n}\right)^{T} M Z_{n}}{H\left(\hat{\Phi}_{n}\right)^{T} M H\left(\hat{\Phi}_{n}\right)}, \quad C_{2}\left(\hat{\Phi}_{n}, Z_{n}\right)=\frac{\left(1-H\left(\hat{\Phi}_{n}\right)\right)^{T} M Z_{n}}{\left(1-H\left(\hat{\Phi}_{n}\right)\right)^{T} M\left(1-H\left(\hat{\Phi}_{n}\right)\right)} .
$$

Finally, we denote by $\Lambda_{n}$ the discrete form of the single layer distribution associated with the computed level set $\hat{\Phi}_{n^{-}}$approximated as in (26) - and multiplied by the gain factor $\lambda$. The observer correction performed as an additional splitting step reads

$$
M \frac{\hat{U}_{n, 3}-\hat{U}_{n, 2}}{\Delta t}=-\Lambda_{n}\left(\left(Z_{n}-C_{1}\left(\hat{\Phi}_{n}\right)\right)^{2}-\left(Z_{n}-C_{2}\left(\hat{\Phi}_{n}\right)\right)^{2}\right) .
$$

We point out that we choose to rely on an explicit time step here due to the non-linearity in the feedback term. Clearly, an implicit time step would ease the stability analysis at the time-discrete level, but at the cost of computing the tangent of the feedback, i.e. the discrete counterpart of (17). Here we have observed that the stability brought by the implicit treatment of the diffusion is sufficient for relying on explicit steps for the other non-linear terms, in particular for the feedback, provided the gain is not too large as shown in our numerical investigation in Section 4. A more detailed analysis will be proposed in a forthcoming paper focusing on discretization aspects of this observer and convergence analysis.

Note that, generally speaking, numerous observers have been time-discretized with splitting methods, which allows to consider their implementation in a generic computational framework as that proposed by the Verdandi Library [21]. This is the case of the discrete-time Kalman Filter considered as a discretization of the continuous-time Kalman Filter [61]. This was also proposed for Luenberger observers in [23]. Moreover, we emphasize that in our case a major advantage lies in the fact that the splitting step is explicit as is already the case for the non-linear reaction term.

Another important aspect in the time-discretization of an observer is the ability to handle the time-sampling of the data, which in practice is imposed and can be quite different from the time-step used in the model. For instance in cardiac electrophysiology, ECGI devices typically produce isochrones with a time-sampling of $\Delta T \in[1,10] \mathrm{ms}$, whereas the simulation time step $\Delta t$ can typically reach $0.1 \mathrm{~ms}[26,70]$. In order to handle the data time-sampling, two strategies are conceivable, namely, we can either use the data only when they are available, or we can rely on some time-interpolation [23]. In the latter case, as the fronts data do not easily lend themselves to interpolation we propose to follow [63] and interpolate (linearly) the corrections, leading to

$$
\begin{aligned}
& M \frac{\hat{U}_{n, 3}-\hat{U}_{n, 2}}{\Delta t}=-\omega_{n, j} \Lambda_{n}\left(\left(Z_{j}-C_{1}\left(\hat{\Phi}_{n}, Z_{j}\right)\right)^{2}-\left(Z_{j}-C_{2}\left(\hat{\Phi}_{n}, Z_{j}\right)\right)^{2}\right) \\
& \quad-\left(1-\omega_{n, j}\right) \Lambda_{n}\left(\left(Z_{j+1}-C_{1}\left(\hat{\Phi}_{n}, Z_{j+1}\right)\right)^{2}-\left(Z_{j+1}-C_{2}\left(\hat{\Phi}_{n}, Z_{j+1}\right)\right)^{2}\right) .
\end{aligned}
$$

where $\left\{t_{j}, t_{j+1}\right\}$ are the data sampling times adjacent to the simulation time $t_{n}$ and

$$
\omega_{n, j}=\frac{t_{j+1}-t_{n}}{t_{j+1}-t_{j}} .
$$




\subsection{Parameter estimation}

We can now present the additional level of parameter identification compatible with our state estimator. To that purpose, we follow the strategy proposed in [62] and further developed in [61]. In essence, this assumes that an effective state observer is already available, as in our case, indeed. The uncertainties on the system trajectory are thus controlled by the state observer, hence, the overall uncertainties can then be considered to be reduced to the parameter space, where a Kalman-based filter is designed. More precisely, a Reduced-Order Extended Kalman Filter (ROEKF) was originally proposed in [62], and a more convenient Reduced-Order Unscented Kalman Filter (ROUKF) was subsequently introduced in [61]. The main advantage of ROUKF is that, unlike ROEKF, it does not require differentiating the model and the discrepancy with respect to the trajectory, but instead relies on "particles" surrounding the trajectory in order to compute the system sensitivities to the data and to the uncertainties. Therefore, the only requirement in ROUKF is to be able to define at the discrete level a full time discretization from one time step to the next, typically in the form

$$
\hat{U}_{n+1}=A_{n+1 \mid n}^{\text {Luen }}\left(\hat{U}_{n}, \theta_{n}, Z_{n}\right),
$$

where $A_{n+1 \mid n}^{\text {Luen }}$ is the discretized dynamics between two consecutive time steps of the state observer, as presented in the previous section. Moreover, ROUKF is based on an underlying least square functional. Here, noting that the functional (25) can be rewritten in the form

$$
\mathcal{J}_{\hat{u}}=\int_{\mathcal{B}}\left(H\left(\phi_{\hat{u}}\right)\left(z-C_{1}\left(\Omega_{\hat{u}}^{\text {in }}\right)\right)+\left(1-H\left(\phi_{\hat{u}}\right)\right)\left(z-C_{2}\left(\Omega_{\hat{u}}^{\text {in }}\right)\right)\right)^{2} d \mathcal{B}=\int_{\mathcal{B}} D(z, \hat{u})^{2} d \mathcal{B}
$$

where the discrepancy reads

$$
D(z, \hat{u})=H\left(\phi_{\hat{u}}\right)\left(z-C_{1}\left(\Omega_{\hat{u}}^{\text {in }}\right)\right)+\left(1-H\left(\phi_{\hat{u}}\right)\right)\left(z-C_{2}\left(\Omega_{\hat{u}}^{\text {in }}\right)\right),
$$

we can introduce at the discrete level the discrete discrepancy

$$
\begin{aligned}
D\left(Z_{n+1}, \hat{U}_{n+1}\right)=H\left(\hat{\Phi}_{n+1}\right)\left(Z_{n+1}-\right. & \left.C_{1}\left(\hat{\Phi}_{n+1}, Z_{n+1}\right)\right) \\
& +\left(1-H\left(\hat{\Phi}_{n+1}\right)\right)\left(Z_{n+1}-C_{2}\left(\hat{\Phi}_{n+1}, Z_{n+1}\right)\right)
\end{aligned}
$$

which is perfectly compatible with the discrepancy definition required in [61]. The full joint state and parameter estimator is then given by [61] and implemented in the Verdandi data assimilation library [21] - see also the documentation of the library [18].

Remark 1 (Internal variables). In many cases, the fully discrete state equation cannot be summarized in a form as simple as (28), due to the presence of internal variables, as e.g. for ionic variables in the example of electrophysiology modeling. In such cases, the state variable needs to incorporate the internal variables in the form $\left(\begin{array}{c}U \\ W\end{array}\right)$, and the dynamical operator accordingly pertains to this complete state variable. Nevertheless, ROUKF naturally allows to deal with internal variables in a very straightforward manner, see [22] for more detail. 
Remark 2 (Ensemble Kalman Filtering (EnKF) alternatives). The UKF filters family is very close in spirit to the Ensemble Kalman filters family [36], but usually with a smaller number of particles aiming at creating a minimal stencil to catch the non-linear operator variations. We believe that a reduced-order EnKF reduced to the parameter space - typically based on square root versions of the EnKF [81], as was done for UKF in [61, 60] - could certainly be an alternative to our choice of ROUKF.

It is clear that the efficiency of the identification method is conditioned by the amount of parameter information that can be extracted from the data. In other words, the question is to determine which parameter can be uniquely recovered by using the output observations, namely, the identifiability as reviewed in [65] for data assimilation, or the structural identifiability of [8]. For the joint state and parameter strategy proposed here, it was shown in $[89,62]$ that, typically, a persistent excitation condition should be satisfied to ensure the convergence of the estimator, at least for small errors. This persistent excitation condition can be seen as an identifiability condition that remains to be proved and related to existing identification results for reaction-diffusion systems with observed propagating fronts. This goes far beyond the scope of this article, but some recent works have investigated e.g. how a traveling front velocity contains the necessary information to recover some parameters - namely, a reaction parameter, with future work announced on the diffusion parameter case [32].

\section{Numerical illustrations and assessments}

In this section we present some numerical illustrations and assessments of our aboveproposed observer strategies. The specific models and data considered here concern cardiac electrophysiology, of which we start by providing a brief summary.

\subsection{Cardiac electrophysiology}

Cardiac electrophysiology aims at describing the electro-chemical phenomena that take place in the cardiac tissue and trigger the muscle mechanical contraction. Due to frequent associated pathologies - such as for example atrial fibrillation or ventricular bradycardia and tachycardia - that directly affect this source of muscle contraction, the study of electrophysiology is crucial for medical applications. Over the past decades, cardiac electrophysiological modeling has made huge progress, and very realistic models and simulations thereof have been obtained based on equations of reaction-diffusion type, see e.g. [25, 42, 28, 27, 80, 39], including for pathological cases [77, 47, 12]. The most widely accepted underlying reaction-diffusion equations are known as the bidomain model and an approximation thereof named monodomain model [83, 76, 49, 27].

The bidomain model equations can be written - in terms of the extracellular potential $u_{e}$, and the transmembrane potential $V_{m}=u_{i}-u_{e}$, with $u_{i}$ the intracellular potential as

$$
\left\{\begin{array}{cc}
A_{m}\left(C_{m} \partial_{t} V_{m}+I_{\text {ion }}\left(V_{m}, \cdots\right)\right)-\operatorname{div}\left(\overrightarrow{\vec{\sigma}}_{i} \cdot \vec{\nabla} V_{m}\right) & \\
=\operatorname{div}\left(\overrightarrow{\vec{\sigma}}_{i} \cdot \vec{\nabla} u_{e}\right)+A_{m} I_{a p p}, & \text { in } \mathcal{B} \times(0, T), \\
\operatorname{div}\left(\left(\overrightarrow{\vec{\sigma}}_{i}+\overrightarrow{\vec{\sigma}}_{e}\right) \cdot \vec{\nabla} u_{e}\right)=-\operatorname{div}\left(\overrightarrow{\vec{\sigma}}_{i} \cdot \vec{\nabla} V_{m}\right), & \text { in } \mathcal{B} \times(0, T),
\end{array}\right.
$$


with adequate boundary conditions

$$
\begin{cases}\left(\overrightarrow{\vec{\sigma}}_{i} \cdot \vec{\nabla} u_{e}\right) \cdot \vec{n}=-\left(\overrightarrow{\vec{\sigma}}_{i} \cdot \vec{\nabla} V_{m}\right) \cdot \vec{n}, & \text { in } \partial \mathcal{B} \times(0, T), \\ \left(\overrightarrow{\vec{\sigma}}_{e} \cdot \vec{\nabla} u_{e}\right) \cdot \vec{n}=0, & \text { in } \partial \mathcal{B} \times(0, T),\end{cases}
$$

where $A_{m}$ is a positive constant denoting the ratio of membrane area per unit volume, $C_{m}$ the membrane capacitance per unit surface and $I_{a p p}$ a given applied stimulus current. The extra-cellular potential $u_{e}$ is defined up to a constant, hence, the condition $\int_{\mathcal{B}} u_{e}=0$ is added in order to have a well-posed problem. In this model the reaction term is given by $I_{i o n}\left(V_{m}, \cdots\right)$, which represents the ionic current across the cellular membrane that separates the intra- and extracellular domains. There is a large variety of ionic models devised to describe this complex process, with a varying number of additional variables entering the function $I_{i o n}\left(V_{m}, \cdots\right)$, the dynamics of these variables being governed by a set of ordinary differential equations. The existence and uniqueness of solutions of the bidomain model equations have been studied for several ionic models in the literature, see for example $[28,9,14,84]$. The typical behavior of a physiological solution is as follows. Initially, the whole domain is with $V_{m}$ at the resting potential around $-80 \mathrm{mV}$. Due to an external stimulus, a depolarization wave occurs, under which the transmembrane potential quickly changes to a value around $20 \mathrm{mV}$. This state remains quite stable for some time - this is called the plateau phase - over which the mechanical contraction occurs. Then follows a repolarization phenomenon, during which cells are in a so-called refractory phase, meaning that a new stimulus would momentarily be unable to trigger a new depolarization.

In our case, we will consider a surface-based bidomain model specially derived for thin structures - such as the atria, namely, the upper chambers of the heart - proposed and mathematically justified in [20] based on an asymptotic analysis, with realistic simulations of atrial electrophysiology given in [29]. This surface-based model is used in this case with the Courtemanche-Ramirez-Nattel ionic model [31], as is generally done when atria are concerned.

Prior to the above modeling case representative of a realistic electrophysiology simulation, we will also employ a simpler model for faster assessment purposes. Namely, we will consider the monodomain model - derived from the bidomain model under the assumption that the intra- and extracellular conductivity tensors are proportional to each other, see $[49,69]$ - in a 1D spatial domain. The monodomain model reads

$$
\left\{\begin{aligned}
A_{m}\left(C_{m} \partial_{t} V_{m}+I_{i o n}\left(V_{m}, w\right)\right)-\operatorname{div}\left(\overrightarrow{\vec{\sigma}}_{m} \cdot \vec{\nabla} V_{m}\right) & =A_{m} I_{a p p}, & & \text { in } \mathcal{B} \times(0, T), \\
\left(\overrightarrow{\vec{\sigma}}_{m} \cdot \vec{\nabla} V_{m}\right) \cdot \vec{n} & =0, & & \text { in } \partial \mathcal{B} \times(0, T) .
\end{aligned}\right.
$$

with $\overrightarrow{\vec{\sigma}}_{m}=\frac{\left\|\overrightarrow{\vec{\sigma}}_{e}\right\|}{\left\|\overrightarrow{\vec{\sigma}}_{i}\right\|+\left\|\vec{\sigma}_{e}\right\|} \overrightarrow{\vec{\sigma}}_{i}$, namely, the geometric average of the previous two tensors. We used the monodomain model in conjunction with the ionic current given by the MitchellSchaeffer model [59].

Our estimation trials are devised with the major motivation of obtaining patientspecific simulations, relying on observers using as data the depolarization maps, or equivalently the so-called isochrone maps providing the time of passage of the depolarization front at each point, see an example in Figure 2. Such data can be typically obtained based on so-called electrocardiographic imaging (ECGI), by which an electrode 


\begin{tabular}{ccccc}
\hline \hline $\begin{array}{c}\sigma_{m} \\
\left(\mathrm{~S} . \mathrm{cm}^{-1}\right)\end{array}$ & $\begin{array}{c}A_{m} \\
\left(\mathrm{~cm}^{-1}\right)\end{array}$ & $\begin{array}{c}C_{m} \\
\left(\mathrm{mF} \cdot \mathrm{cm}^{-2}\right)\end{array}$ & $\begin{array}{c}\tau_{\text {in }} \\
\left(\mathrm{cm}^{2} \cdot \mathrm{mA}^{-1}\right)\end{array}$ & $\begin{array}{c}\tau_{\text {out }} \\
\left(\mathrm{cm}^{2} \cdot \mathrm{mA}^{-1}\right)\end{array}$ \\
\hline \hline $2.010^{-3}$ & 1 & 1 & 0.6 & 6 \\
\hline \hline$\tau_{\text {open }}$ & $\tau_{\text {close }}$ & $V_{\min }$ & $V_{\max }$ & $V_{\text {gate }}$ \\
$(\mathrm{ms})$ & $(\mathrm{ms})$ & $(\mathrm{mV})$ & $(\mathrm{mV})$ & $(\mathrm{mV})$ \\
\hline \hline 120 & 300 & 0 & 1 & 0.13 \\
\hline
\end{tabular}

Table 1: 1D monodomain model parameters
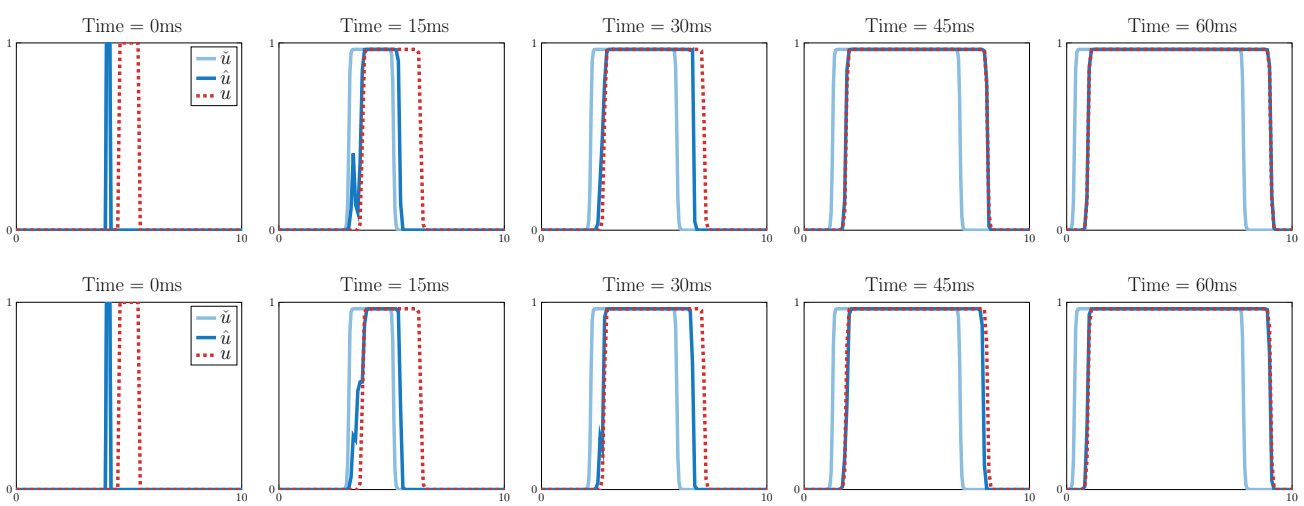

Figure 1: State observer assessment for 1D model: reference solution in dashed red, observer in dark blue, direct simulation without observer correction in light blue - Top row shows estimation with complete data, and bottom row with sampled data

vest records the potential at various points on the body surface and an inverse method allows to reconstruct the depolarization maps, see e.g. [70].

\subsection{State estimation}

\subsubsection{D monodomain model}

Let us first consider here the 1D monodomain equations coupled with the MitchellSchaeffer model. The model parameters are listed in Table 1, with the classical notation for the Mitchell-Schaeffer model as e.g. used in [20], and no external current is applied. We aim at using the above-proposed observer methodology to circumvent an error in the initial condition based on data associated with the location of the reference propagation front. We use a standard centered finite difference scheme for space discretization, with the Dirac function treatment and the time scheme discussed in Section 3.4. The corresponding numerical simulations have been performed within Matlab. The $10 \mathrm{~cm}$ domain is split into 1,000 cells, and the time step is $1 \mathrm{~ms}$. The numerical results are shown in Figure 1 in two cases: 1- when the propagation front is provided at every simulation time step, and 2- when the propagation front is available only with a given - quite coarse - time sampling (seven equally-spaced times in the simulation window), in which case the discrepancy operator is interpolated at all other time steps as explained in Section 3.4 above. We can see that the proposed observer is very effective in both cases. Concerning the oscillation that appears in one snapshot (at time $15 \mathrm{~ms}$ with complete data), 

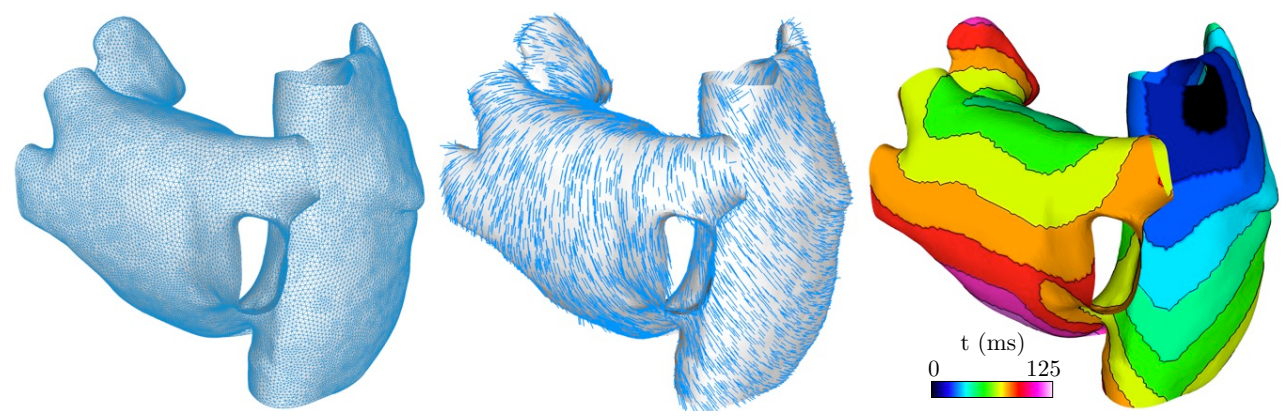

Figure 2: Anatomical model of the atria, with computational mesh (left), fiber directions on mid-surface (center), and isochrones produced by reference physiological simulation (right)

we conjecture that this is due to the discretization of the Dirac function, for which some improvements may be sought.

\subsubsection{Bidomain atrial model}

The surface domain considered - corresponding to an anatomical model of cardiac atria - is visualized in Figure 2, together with the isochrones produced by the reference solution, namely, the data that will be used in the observer. This anatomical model is described in greater detail in [29], where all the modeling parameters used to obtain a physiological solution are also specified, including for the direction of the muscle fibers and angular variations thereof between epi- and endocardium - see central part of the figure, and the surface mesh features about 22,250 vertices. Spatial discretization is performed with P1-Lagrange elements, with again the Dirac function treatment and the time scheme discussed in Section 3.4. For the implementation, we rely on $F E L i S c E^{1}$, a finite element library developed at Inria and dedicated to life sciences and engineering problems, where the parallelism is based on the PETSc library [6]. With a simulation time step of $0.1 \mathrm{~ms}$, this leads to direct simulation times of about 4 minutes on a standard multi-core workstation. Such relatively modest simulation times - for a bidomain model - are only allowed by the fact that a surface model is considered, of course. Note that this simulation time could be further improved by using mesh adaptation around the front in the spirit of [33]. As mentioned in Section 3.4, this should also allow to directly compute the weak form of the feedback correction supported by the observer front without relying on Dirac approximations.

Figure 3 shows the simulation results. The observer is started with an initial condition that significantly differs - in location - from that of the reference solution. Namely, the initial potential is shifted, which could typically be due to an uncertainty in the location of the sino-atrial node. Nevertheless, the observer state is very effectively corrected, as the estimated state is very close to the reference for times greater than $50 \mathrm{~ms}$, which can be verified in Figure 4 where the absolute errors are plotted in time. By contrast, the direct simulation starting from the same initial condition as the observer and without any correction maintains a significant discrepancy with the reference throughout the simulation, see Figure 3.

\footnotetext{
${ }^{1}$ http://felisce.gforge.inria.fr
} 


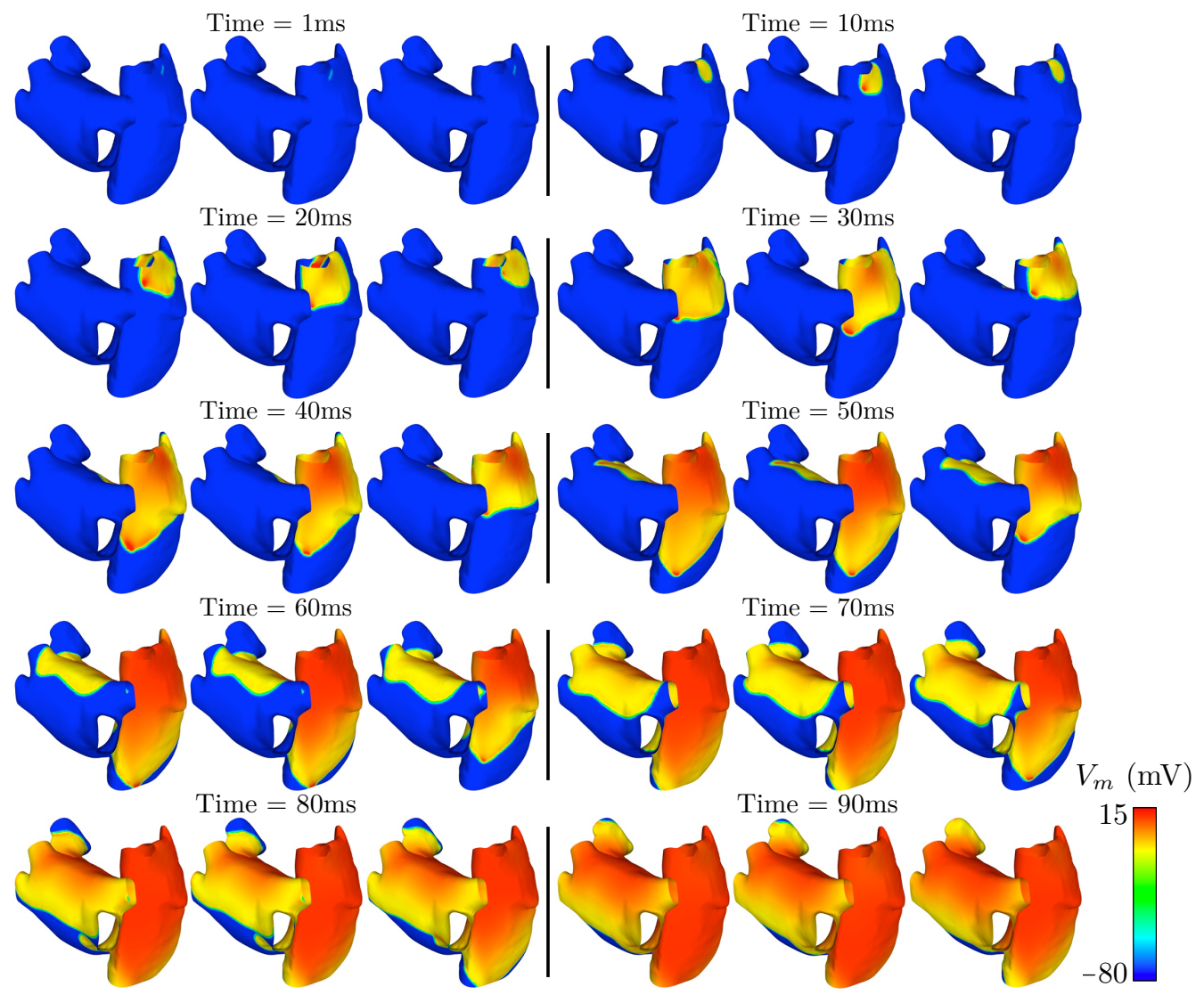

Figure 3: Comparison of observer (left), reference (center) and direct simulation (right) at successive time steps

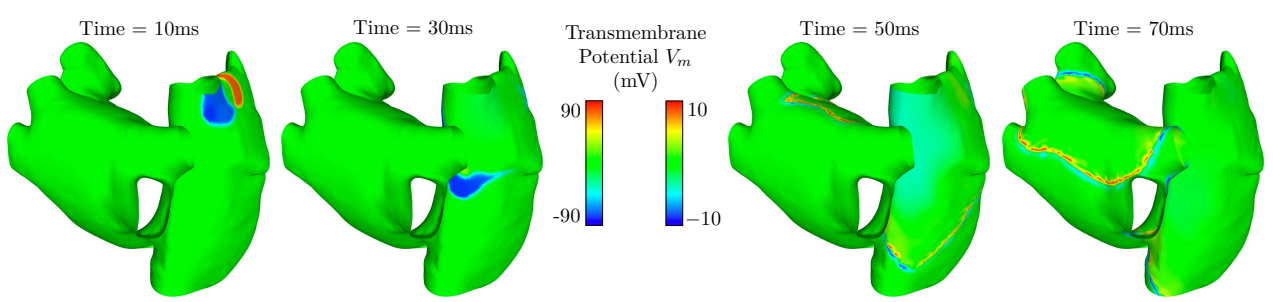

Figure 4: Error between observer and reference solutions at successive time steps (note that a much narrower color map is used in the last two to better reveal the error) 

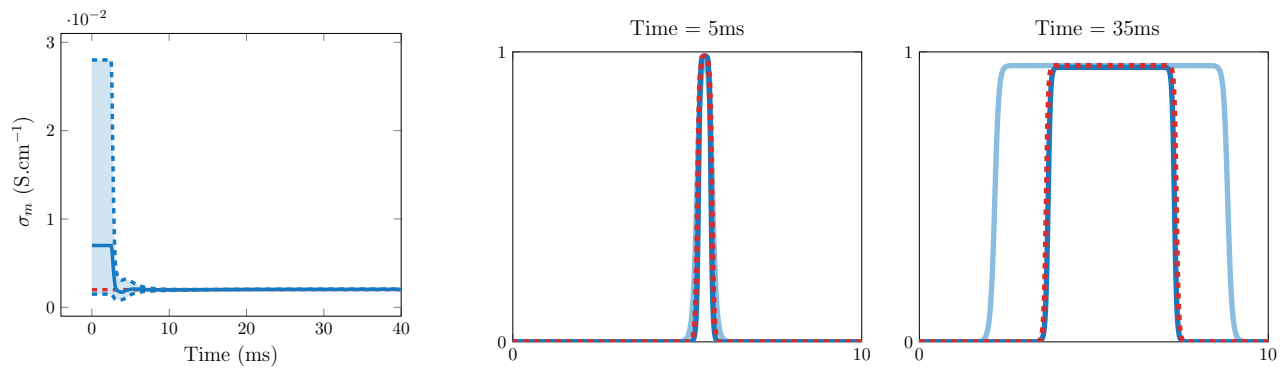

Figure 5: Estimated parameter with estimated standard deviation band (left, where dashed red represents reference value) - Comparison of reference solution in dashed red, observer in dark blue, direct simulation without observer correction in light blue at two successive time steps (center and right)

\subsection{Joint state-parameter estimation}

We now turn our attention to joint state-parameter estimation, and again consider $1 \mathrm{D}$ and atrial test cases to assess the ability of our strategy to cope with errors present both in the initial condition and in the physical parameters.

\subsubsection{D monodomain model}

The estimated parameter is the electrical conductivity $\sigma_{m}$, assumed to be homogeneous in this case. In the first scenario we start the observer - and a direct simulation - from the reference initial condition, albeit with an altered parameter. Figure 5 shows that the combined observer very accurately (and quickly) estimates the parameter value while effectively preserving the correct solution profile. By contrast, the altered conductivity parameter drives the direct simulation increasingly further away from the reference.

In the second scenario, we start the observer and direct simulation with both an altered conductivity parameter and a shifted initial condition. In this case, we can see in Figure 6-top that the joint state-parameter observer fails to estimate the parameter value, hence, is also unable to effectively track the depolarization propagation. This is a classical difficulty in Kalman-based estimation approaches in their extensions to nonlinear problems. In particular, here, the parameter corrections are based on computing sensitivities in the observed quantity, and such sensitivities are not meaningful when the observer state notably differs from the reference state, which is the case due to the initial condition error. A practical interpretation in Figure 6-top is that the propagation front in the observer is early - compared to the reference - on one side, which would require a decrease in the conductivity parameter to compensate, albeit late on the other side, which induces a conflicting sensitivity. To circumvent this difficulty, one standard practical strategy consists in delaying the startup of the parameter estimation stage, while letting the state observer start tracking the reference [62]. This is very effective in our case as well, as seen in Figure 6-bottom.

\subsubsection{Bidomain atrial model}

We conclude our assessments with joint state-parameter estimation trials in the atrial electrophysiology model. We now have two estimated parameter values, namely, the 

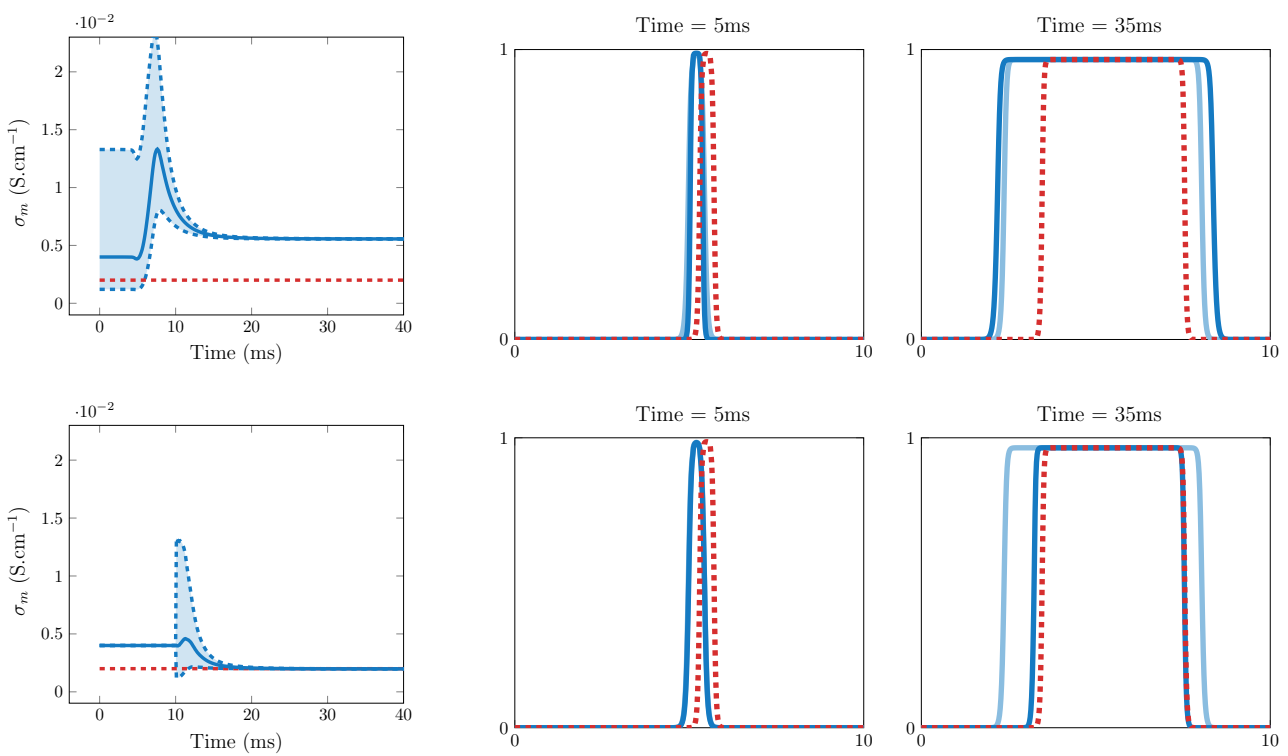

Figure 6: Estimated parameter with estimated standard deviation band (left, where dashed red represents reference value) - Comparison of reference solution in dashed red, observer in dark blue, direct simulation without observer correction in light blue at two successive time steps (center and right) - Top row for direct startup of joint state-parameter estimation, bottom row for delayed startup of parameter estimation stage
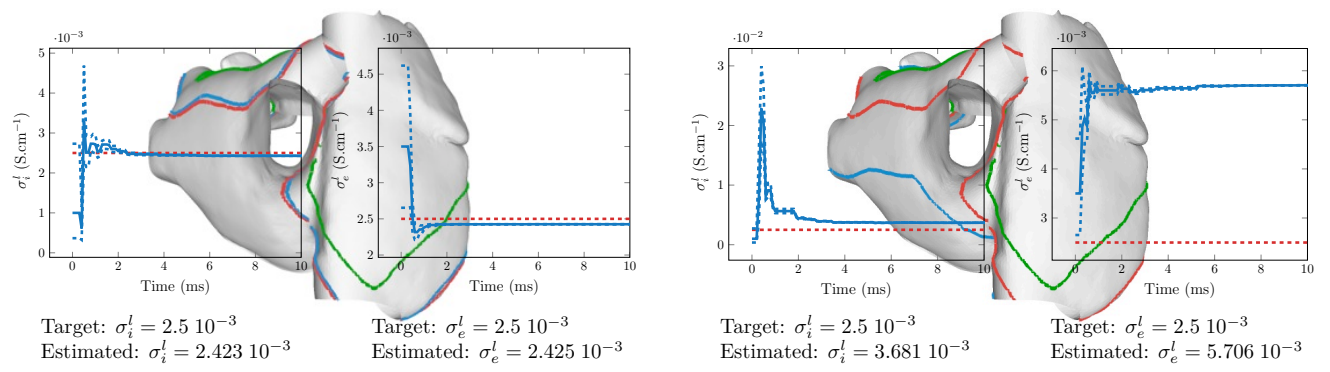

Figure 7: Estimation of two conductivity parameters with (left) and without (right) initial condition error - Reference parameter values in dashed red, and bounds of estimated standard deviation interval in dashed blue - Propagation fronts are shown in the background at time $70 \mathrm{~ms}$ (red, blue and green for the reference, observer and direct simulation, respectively) 

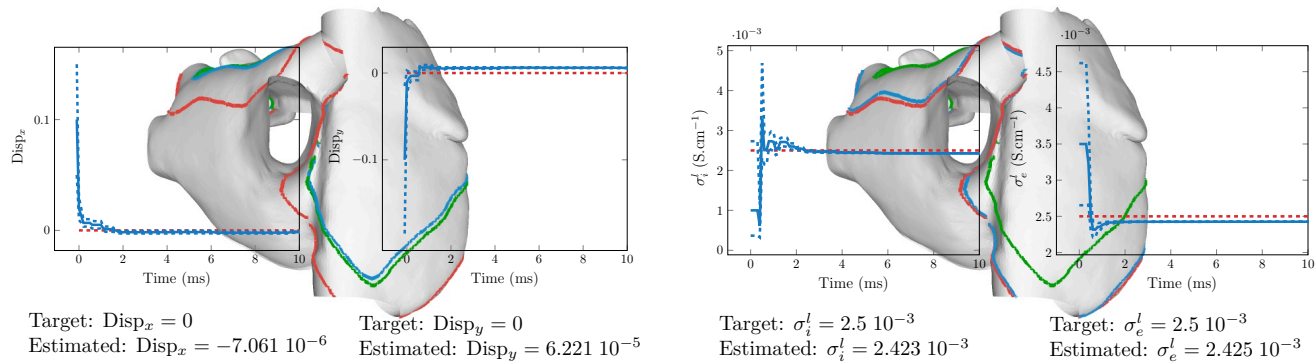

Figure 8: First estimation run for initial condition location (left), then second estimation run for conductivity parameters (right) - Reference parameter values in dashed red, and bounds of estimated standard deviation interval in dashed blue - Propagation fronts are shown in the background at time $70 \mathrm{~ms}$ (red, blue and green for the reference, observer and direct simulation, respectively)

intra- and extracellular conductivities along the fiber direction, denoted by $\sigma_{i}^{l}$ and $\sigma_{e}^{l}$, respectively.

In the first scenario (Figure 7-left) we start the observer and direct simulation with altered parameter values, albeit with the exact initial condition. The joint estimation strategy is very effective in this case, as in the above $1 \mathrm{D}$ trial.

In the second scenario, we incorporate an error in the initial condition - in addition to the errors in the parameters - in the form of a shift in the location of the initial potential, as in the above state estimation trial. In this case, the standard joint estimation fails as in the 1D model (Figure 7-right). Rather than resorting to a startup delay in the parameter estimation as before, we will illustrate another - more powerful strategy that consists in parametrizing the initial condition location and estimating these initial condition parameters in a first run of a joint-state parameter observer, prior to estimating the conductivities in a second run. In our case we parametrize - according to the specific uncertainty considered - by two scalar quantities corresponding to shifts along the surface. Note that this strategy presents the additional benefit of providing actual information on the initial condition, which is very valuable in medical applications where a pathology in the electrical activation onset may be sought, e.g. with an ectopic focus or reentry phenomena [68]. This strategy is demonstrated in Figure 8, where we see the results of the initial condition location estimation run on the left-hand side, and of the second run with conductivity estimation on the right-hand side. In the first run the initial condition location is quite accurately retrieved, but the observer propagation diverges from the reference - quite like the direct simulation - due to the error in the conductivity parameters. Note that this error does not hinder the initial condition estimation, which is effectively performed in a very short moment after the activation onset, hence does not see the later propagation divergence. Then, in the second run parameter estimation is performed on the conductivity parameters only, and the results show that the joint state-parameter estimation is now very effective.

Concerning the cost of our estimator, we can evaluate the additional simulation time with respect to the reference simulation. The state estimation introduces an additional simulation cost of about $5 \%$. Concerning the parameter estimation, each particle is sent in parallel and communicates at the end of each forward time step. However, in the forward time step of each particle the diffusion operator must be re-assembled as the 
estimated diffusion coefficient has changed. A simulation where the diffusion operator is re-computed at each time step is about 10 times as long as a direct simulation where the diffusion operator is pre-assembled and factorized only at the beginning of the simulation. In addition, the parameter estimation communications induce a $30 \%$ time overhead. This should be compared to a $4 \mathrm{D}$-Var parameter estimation where the diffusion operator is in fact pre-assembled and factorized, but at each iteration of the gradient descent. Therefore, a standard 4D-Var should typically converge in less than 13 iterations - or 26 when considering two heart beats in the sequential estimation - to compete with our estimator. We also point out that, in a completely realistic electrophysiological case, the domain is moving due to heart contraction, which imposes to systematically re-assemble the operators at each time step.

\section{Concluding remarks}

We have proposed a Luenberger observer for reaction-diffusion models with propagating front features, and for data associated with the location of the front over time. This can be valuable in electrophysiology when ECGI data are available, and thus represents an alternative to performing estimation directly based on ECG measurements as considered in [30] where Kalman filtering is used due to the difficulty of devising a Luenberger observer with this type of data. Our observer formulation was based on the eikonal-curvature equation that can be derived from the reaction-diffusion equation with an asymptotic analysis, and that directly represents the propagation of the front itself, via a level set representation. We started by proposing an observer for this associated eikonal-curvature equation, drawing our inspiration from image processing methods. We then carried over this observer to the underlying reaction-diffusion equation by an "inverse asymptotic analysis", namely, by ensuring that an asymptotic analysis of the final observer - for the reaction-diffusion model - yields the observer designed for the eikonalcurvature equation. We also discussed how - once such an adequate state observer has been formulated - extensions to joint state-parameter estimation can be obtained by using the earlier-proposed ROUKF strategy.

We have then illustrated and assessed our proposed observer strategy - both for state and state-parameter estimation - with test problems associated with electrophysiology modeling. Namely, we have considered a 1D monodomain model, on the one hand, and a surface-based bidomain model with a realistic atrial anatomy - including for the fiber directions - on the other hand. For both models our trials have shown that state estimation is very effective with the proposed Luenberger observer. As concerns joint state-parameter estimation we have been facing difficulties that are quite usual for a Kalman filter used in a nonlinear estimation problem - here for parameter estimation, due to the initial condition error in the state. Consequently we have proposed two methods to circumvent these difficulties, namely, 1- let the state filter operate by itself for some rather short time before starting the parameter estimation, and 2- circumscribe the initial condition error with a first run of state-parameter estimation in which the estimated parameters characterize the initial condition location. Both approaches have proven to be successful, the second one featuring the additional benefit of providing actual information on the initial condition.

Finally, we conjecture that our observer strategy could be used with comparable effectiveness with other reaction-diffusion models of propagating type. Other types of 
equations such as wave equations can also display front propagation phenomena and may be studied from a front-based asymptotic point of view [75]. Our shape-based data fitting term could also lead to original observer formulations in such cases. Further perspectives include resorting to topological gradient instead of - or in addition to - shape gradient in the observer design to deal with changes of topology in the fronts [15], and the use of several front levels in the data in association with a higher-order asymptotic analysis and a multiphase level set strategy [85], in particular.

Acknowledgment: The research leading to these results has received partial funding from the European Union's Seventh Framework Programme for research, technological development and demonstration, under grant agreement \#611823 (VP2HF Project).

[1] R.A. Anthes. Data assimilation and initialization of hurricane prediction model. Journal of the Atmospheric Sciences, 31:702-719, 1974.

[2] D.G. Aronson and H.F. Weinberger. Multidimensional nonlinear diffusion arising in population genetics. Advances in Mathematics, 30:33-76, 1978.

[3] U.M. Ascher, S.J. Ruuth, and B.T.R. Wetton. Implicit-explicit methods for time-dependent partial differential equations. SIAM Journal on Numerical Analysis, 1995.

[4] M.I. Asensio and L. Ferragut. On a wildland fire model with radiation. International Journal for Numerical Methods in Engineering, 54(1):137-157, 2002.

[5] D. Auroux and J. Blum. Back and forth nudging algorithm for data assimilation problems. Comptes Rendus Mathematique, 340(12):873-878, 2005.

[6] S. Balay, W. D. Gropp, L. Curfman McInnes, and Smith B. F. Efficient management of parallelism in object oriented numerical software libraries. In E. Arge, A. M. Bruaset, and H. P. Langtangen, editors, Modern Software Tools in Scientific Computing, pages 163-202. Birkhäuser Press, 1997.

[7] G. Bellettini, P. Colli Franzone, and M. Paolini. Convergence of front propagation for anisotropic bistable reaction-diffusion equations. Asymptotic Analysis, 15(3-4):325-358, 1997.

[8] R. Bellman and K.J. Aström. On structural identifiability. Mathematical Biosciences, 7(3-4):329$339,1970$.

[9] M. Bendahmane and H.K. Karlsen. Analysis of a class of degenerate reaction-diffusion systems and the bidomain model of cardiac tissue. Networks and Heterogeneous Media, 1:185-218, 2006.

[10] R.P. Beyer and R.J. LeVeque. Analysis of a one-dimensional model for the immersed boundary method. SIAM Journal on Numerical Analysis, 1992.

[11] J. Blum, F.-X. Le Dimet, and I.M. Navon. Data assimilation for geophysical fluids. In R. Temam and J. Tribbia, editors, Handbook of Numerical Analysis: Computational Methods for the Atmosphere and the Oceans. Elsevier, 2008.

[12] M. Boulakia, J.-F. Gerbeau, and E. Schenone. Reduced-order modeling for cardiac electrophysiology. Application to parameter identification. International Journal for Numerical Methods in Biomedical Engineering, 28:727-744, 2012.

[13] A.-C. Boulanger, P. Moireau, B. Perthame, and J. Sainte-Marie. Data Assimilation for hyperbolic conservation laws. A Luenberger observer approach based on a kinetic description. Communications in Mathematical Sciences, 13(3):587 - 622, 2015.

[14] Y. Bourgault, Y. Coudière, and C. Pierre. Existence and uniqueness of the solution for the bidomain model used in cardiac electrophysiology. Nonlinear Analysis-Real World Applications, 10(1):458482, 2009.

[15] M. Burger, B. Hackl, and W. Ring. Incorporating topological derivatives into level set methods. Journal of Computational Physics, 194(1):344-362, 2004.

[16] M. Cencini, C. Lopez, and D. Vergni. Reaction-diffusion systems: front propagation and spatial structures. In The Kolmogorov Legacy in Physics - Lecture Notes in Physics, pages 187-210. Springer, 2003.

[17] T.F. Chan and L.A. Vese. Active contours without edges. IEEE Transactions on Image Processing 10(2):266-277, 1991.

[18] D. Chapelle, K. Charpentier, N. Claude, M. Fragu, V. Mallet, P. Moireau, and C. Mouton. Documentation for Verdandi 1.6. Inria, verdandi.gforge.inria.fr/doc-1.6/verdandi-1.6.pdf.

[19] D. Chapelle, N. Cîndea, M. De Buhan, and P. Moireau. Exponential convergence of an observer based on partial field measurements for the wave equation. Mathematical Problems in Engineering, 2012, 2012. 
[20] D. Chapelle, A. Collin, and J.-F. Gerbeau. A surface-based electrophysiology model relying on asymptotic analysis and motivated by cardiac atria modeling. M3AS, 23(14):2749-2776, 2013.

[21] D. Chapelle, M. Fragu, V. Mallet, and P. Moireau. Fundamental principles of data assimilation underlying the Verdandi library: applications to biophysical model personalization within euHeart. Medical $\&$ Biological Eng $\&$ Computing, pages 1-13, 2012.

[22] D. Chapelle, A. Gariah, P. Moireau, and J. Sainte-Marie. A Galerkin strategy with Proper Orthogonal Decomposition for parameter-dependent problems - Analysis, assessments and applications to parameter estimation. ESAIM: Mathematical Modelling and Numerical Analysis, 47(6):1821-1843, 2013.

[23] N. Cîndea, A. Imperiale, and P. Moireau. Data assimilation of time under-sampled measurements using observers, application to wave-like equations. ESAIM: Control, Optimisation and Calculus of Variations, 2014. in press.

[24] T. Colin, A. Iollo, Lombardi. D, and O. Saut. System identification in tumor growth modeling using semi-empirical eigenfunctions. M3AS, 2(1):137-157, 2012.

[25] P. Colli Franzone, L. Guerri, and S. Rovida. Wavefront propagation in an activation model of the anisotropic cardiac tissue: asymptotic analysis and numerical simulations. Journal of Mathematical Biology, 28:121-176, 1990.

[26] P. Colli Franzone and L.F. Pavarino. A parallel solver for reaction-diffusion systems in computational electrocardiology. Mathematical Models and Methods in Applied Sciences, 14(6):883-911, June 2004

[27] P. Colli Franzone, L.F. Pavarino, and B. Taccardi. Simulating patterns of excitation, repolarization and action potential duration with cardiac bidomain and monodomain models. Mathematical Biosciences, 197(1):35-66, 2005.

[28] P. Colli Franzone and G. Savaré. Degenerate evolution systems modeling cardiac electric field at micro and macroscopic. Evolution Equations, Semigroups and Functional Analysis. Progress in Nonlinear Differential Equations and Their Applications, 50:49-78, 2002.

[29] A. Collin, J.-F. Gerbeau, M. Hocini, M. Haïssaguerre, and D. Chapelle. Surface-based electrophysiology modeling and assessment of physiological simulations in atria. FIMH 2013, 7945:352-359, 2013.

[30] C. Corrado, J.-F. Gerbeau, and P. Moireau. Identification of weakly coupled multiphysics problems. Application to the inverse problem of electrocardiography. Journal of Computational Physics, 283:271-298, 2015.

[31] M. Courtemanche, R.J. Ramirez, and S. Nattel. Ionic mechanisms underlying human atrial action potential properties: insights from a mathematical model. American Journal of Physiology, 275(1 Pt 2):H301-H321, 1998.

[32] M. Cristofol, I. Kaddouri, G. Nadin, and L. Roques. Coefficient determination via asymptotic spreading speeds. Inverse Problems, 30(3):035005, February 2014

[33] C. Dapogny, C. Dobrzynski, and P. Frey. Three-dimensional adaptive domain remeshing, implicit domain meshing, and applications to free and moving boundary problems. Journal of Computational Physics, 262:358 - 378, 2014

[34] M.C. Delfour and J.-P. Zolésio. Shapes and Geometries: Analysis, Differential Calculus, and Optimization (Advances in Design and Control). SIAM, second edition, 2011.

[35] B. Engquist, A.-K. Tornberg, and R. Tsai. Discretization of Dirac delta functions in level set methods. Journal of Computational Physics, 207(1):28-51, 2005.

[36] G. Evensen. Data Assimilation - The Ensemble Kalman Filter. Springer Verlag, 2007.

[37] B. Fabrèges and B. Maury. Approximation of single layer distributions by Dirac masses in finite element computations. Journal of Scientific Computing, 58(1):25-40, May 2013

[38] S.C. Ferreira Jr., M.L. Martins, and M.J. Vilela. Reaction-diffusion model for the growth of avascular tumor. Physical Review E, 65:021907 (8pp), 2002.

[39] S. Göktepe and E. Kuhl. Computational modeling of cardiac electrophysiology: A novel finite element approach. International Journal for Numerical Methods in Engineering, 79(2):156-178, 2009.

[40] P. Haldenwang and D. Pignol. Dynamically adapted mesh refinement for combustion front tracking. Computers \& fluids, 31(4):589-606, 2002.

[41] S. Haney, P. Thompson, T. Cloughesy, J. Alger, and A. Toga. Tracking tumor growth rates in patients with malignant gliomas: A test of two algorithms. Am. J. Neuroradiol., 22(1):73-82, 2001.

[42] D.M. Harrild and S.H. Craig. A computer model of normal conduction in the human atria. Circulation Research, 87:e25-e36, 2000. 
[43] M. Hintermüller and W. Ring. An inexact Newton-C-G-type active contour approach for the minimization of the Mumford-Shah functional. Journal of Mathematical Imaging and Vision, 20:19$42,2004$.

[44] C. Hogea, C. Davatzikos, and G. Biros. An image-driven parameter estimation problem for a reaction-diffusion glioma growth model with mass effects. Journal of Mathematical Biology, 56(6):793-825, 2008.

[45] J. E. Hoke and R. A. Anthes. The initialization of numerical models by a dynamic-initialization technique. Monthly Weather Review, 104(12):1551-1556, 1976.

[46] D. Horstmann. From 1970 until present: the Keller-Segel model in chemotaxis and its consequences. Jahresbericht der Deutschen Mathematiker-Vereinigung, 105:103-165, 2003.

[47] V. Jacquemet. An eikonal approach for the initiation of reentrant cardiac propagation in reactiondiffusion models. IEEE Transactions On Biomedical Engineering, 57(9):2090-2098, 2010.

[48] C.K.R.T. Jones. Stability of the travelling wave solution of the FitzHugh-Nagumo system. Transactions of the American Mathematical Society, 286(2):431-469, 1984.

[49] J. Keener and J. Sneyd. Mathematical Physiology. Springer, 2004.

[50] J.P. Keener. An eikonal-curvature equation for action potential propagation in myocardium. Journal of Mathematical Biology, 29:629-651, 1991.

[51] A.N. Kolmogorov, I.G. Petrovsky, and N.S. Piskunov. Etude de l'équation de la diffusion avec croissance de la quantité de matière et son application à un problème biologique. Mosc. Univ. Bull. Math., 1937.

[52] E. Konukoglu, O. Clatz, B. H. Menze, B. Stieltjes, M.-A. Weber, E. Mandonnet, H. Delingette, and N. Ayache. Image guided personalization of reaction-diffusion type tumor growth models using modified anisotropic eikonal equations. Medical Imaging, IEEE Transactions, 29(1):77-95, 2010.

[53] S. Lakshmivarahan and J.M. Lewis. Nudging methods: A critical overview. In S.K. Park and L. Xu, editors, Data Assimilation for Atmospheric, Oceanic, and Hydrologic Applications, volume XVIII. Springer, 2008

[54] F.-X. Le Dimet and O. Talagrand. Variational algorithms for analysis and assimilation of meteorological observations: theoretical aspects. Tellus A, 38(2):97-110, 1986.

[55] C.D. Levermore and J.X. Xin. Multidimensional stability of traveling waves in a bistable reaction-diffusion equation, II. Communications in Partial Differential Equations, 17(11-12):19011924, 1992.

[56] X.-D. Li and C.-Z. Xu. Infinite-dimensional Luenberger-like observers for a rotating body-beam system. Systems \& Control Letters, 60(2):138-145, 2011.

[57] D.G. Luenberger. An introduction to observers. IEEE Transactions on Automatic Control, 16:596$602,1971$.

[58] J. Mandel, L.S. Bennethum, J.D. Beezley, J.L. Coen, C.C Douglas, L.P. Franca, M. Kim, and A. Vodacek. A wildland fire model with data assimilation. Mathematics and Computers in Simulation, $79(3): 584-606,2008$

[59] C.C. Mitchell and D.G. Schaeffer. A two-current model for the dynamics of cardiac membrane. Bulletin Math. Bio., 65:767-793, 2003.

[60] P. Moireau and D. Chapelle. Erratum of article "Reduced-order Unscented Kalman Filtering with application to parameter identification in large-dimensional systems". ESAIM: Control, Optimisation and Calculus of Variations, 17(2):406-409, 2011.

[61] P. Moireau and D. Chapelle. Reduced-order Unscented Kalman Filtering with application to parameter identification in large-dimensional systems. ESAIM: Control, Optimisation and Calculus of Variations, 17(2):380-405, 2011.

[62] P. Moireau, D. Chapelle, and P. Le Tallec. Joint state and parameter estimation for distributed mechanical systems. Computer Methods in Applied Mechanics and Engineering, 1987(6-8):659-677, 2008.

[63] P. Moireau, D. Chapelle, and P. Le Tallec. Filtering for distributed mechanical systems using position measurements: Perspectives in medical imaging. Inverse Problems, 25(3):035010 (25pp), 2009.

[64] V. Moreau-Villéger, H. Delingette, M. Sermesant, H. Ashikaga, E. McVeigh, and N. Ayache. Building maps of local apparent conductivity of the epicardium with a $2 \mathrm{D}$ electrophysiological model of the heart. IEEE Transactions on Bio-Medical Engineering, 53(8):1457-1466, 2006.

[65] I. M. Navon. Practical and theoretical aspects of adjoint parameter estimation and identifiability in meteorology and oceanography. Dynamics of Atmospheres and Oceans, 27(1-4):55-79, 1998.

[66] I. M. Navon. Data assimilation for numerical weather prediction: A review. In Data Assimilation for Atmospheric, Oceanic and Hydrologic Applications. Springer, 2009. 
[67] S. Osher and R. Fedkiw. Level Set Methods and Dynamic Implicit Surfaces. Applied Mathematical Sciences, 2002.

[68] A. M. Pertsov, J. M. Davidenko, R. Salomonsz, W. T. Baxter, and J. Jalife. Spiral waves of excitation underlie reentrant activity in isolated cardiac muscle. Circulation Research, 72(3):631650, 1993.

[69] M. Potse, B. Dubé, J. Richer, A. Vinet, and R.M. Gulrajani. A comparison of monodomain and bidomain reaction-diffusion models for action potential propagation in the human heart. IEEE Transactions on Biomedical Engineering, 53(12):2425-2435, 2006.

[70] C. Ramanathan, R.J. Ghanem, P. Jia, K. Ryu, and Y. Rudy. Noninvasive electrocardiographic imaging for cardiac electrophysiology and arrhythmia. Nature Medicine, 10:422-428, 2004.

[71] K. Ramdani, M. Tucsnak, and G. Weiss. Recovering the initial state of an infinite-dimensional system using observers. Automatica, 46(10):1616-1625, 2010

[72] J. Rauch and J. Smoller. Qualitative theory of the FitzHugh-Nagumo equations. Advances in Mathematics, 27(1):12-44, 1978.

[73] P.J. Riggan and R.G Tissell. Airborne remote sensing of wildland fires. In A. Bytnerowicz, M. Arbaugh, C. Andersen, and A. Riebau, editors, Wildland Fires and Air Pollution. Developments in Environmental Science, volume 8, chapter 6. The Netherlands: Elsevier, 2009.

[74] M.C. Rochoux, B. Cuenot, S. Ricci, A. Trouvé, B. Delmotte, S. Massart, R. Paoli, and R. Paugam. Data assimilation applied to combustion. Comptes Rendus Mécanique, 341:266-276, 2013.

[75] O. Runborg. Mathematical models and numerical methods for high frequency waves. Communications in Computational Physics, 2(5):827-880, 2007.

[76] F.B. Sachse. Computational Cardiology: Modeling of Anatomy, Electrophysiology and Mechanics. Springer-Verlag, 2004

[77] M. Sermesant, K. Rhode, G.I. Sanchez-Ortiz, 0. Camara, R. Andriantsimiavona, S. Hegde, D. Rueckert, P. Lambiase, C. Bucknall, E. Rosenthal, H. Delingette, D.L.G Hill, N. Ayache, and R. Razavi. Simulation of cardiac pathologies using an electromechanical biventricular model and XMR interventional imaging. Medical Image Analysis, 9(5):467-480, 2005.

[78] J.A. Sethian. Theory, algorithms, and applications of level set methods for propagating interfaces. Acta numerica, 5:309-395, 1996.

[79] D. R. Stauffer and J.-W. Bao. Optimal determination of nudging coefficients using the adjoint equations. Tellus A, 45(5):358-369, 1993.

[80] J. Sundnes, G.T. Lines, X. Cai, B.F. Nielsen, K.A. Mardal, and A. Tveito. Computing the Electrical Activity in the Heart, volume 1 of Monographs in Computational Science and Engineering. SpringerVerlag, 2006.

[81] M. K. Tippett, J. L. Anderson, C. H. Bishop, T. M. Hamill, and J. S. Whitaker. Ensemble square root filters. Monthly Weather Review, 131(7):1485-1490, 2003.

[82] P. Tracqui and M. Mendjeli. Modelling three-dimensional growth of brain tumours from time series of scans. Mathematical Models and Methods in Applied Sciences, 9(04):581-598, 1999.

[83] L. Tung. A bi-domain model for describing ischemic myocardial d-c potentials. PhD thesis, Massachusetts Institute of Technology. Dept. of Electrical Engineering and Computer Science, 1978.

[84] M. Veneroni. Reaction-diffusion systems for the macroscopic bidomain model of the cardiac electric field. Nonlinear Analysis: Real World Applications, 10(2):849-868, 2009.

[85] L.A. Vese and F.C. Chan. A multiphase level set framework for image segmentation using the Mumford and Shah model. International Journal of Computer Vision, 50(3):271-293, 2002

[86] P.A. Vidard, F.-X. Le Dimet, and A. Piacentini. Determination of optimal nudging coefficients. Tellus A, 55(1):1-15, 2003.

[87] R.L. Wahl, L.E. Quint, R.D. Cieslak, A.M. Aisen, R.A. Koeppe, and C.R. Meyer. "Anatometabolic" tumor imaging: fusion of FDG PET with CT or MRI to localize foci of increased activity. Journal of Nuclear Medicine, 34(7):1190-1197, 1993.

[88] J.X. Xin. Multidimensional Stability of Traveling Waves in a Bistable Reaction-Diffusion Equation .1. Communications in Partial Differential Equations, 17(11-12):1889-1899, 1992.

[89] Q. Zhang. Adaptive observer for mimo linear time varying systems,. IEEETransactions on Automatic Control, 3:525-529, 2002.

[90] H.K. Zhao, T. Chan, B. Merriman, and S. Osher. A variational level set approach to multiphase motion. Journal of Computational Physics, 127(1):179-195, 1996.

[91] X. Zou, I.M. Navon, and F.-X. Le Dimet. An optimal nudging data assimilation scheme using parameter estimation. Quarterly Journal of the Royal Meteorological Society, 118(508):1163-1186, 1992 


\section{Appendix}

Proposition 4. Defining

$$
J_{\tau}=\int_{\Gamma_{\tau}} \mathcal{F}\left(\tau, \Gamma_{\tau}, \vec{x}\right) d \Gamma
$$

and assuming that the closed contour $\Gamma_{\tau}$ is displaced with the flow field $\vec{\psi}$, we have

$$
\left.\frac{d J_{\tau}}{d \tau}\right|_{\tau=0}=\int_{\Gamma_{0}}\left(\dot{\mathcal{F}}-\vec{\nabla}_{\Gamma} \mathcal{F} \cdot \vec{\psi}+\kappa \mathcal{F} \vec{\psi} \cdot \vec{n}\right) d \Gamma,
$$

where $\vec{\nabla}_{\Gamma}$ denotes the tangential gradient, $\kappa$ the additive curvature, $\vec{n}$ the unit normal vector, and $\dot{\mathcal{F}}$ is the particle derivative, namely,

$$
\dot{\mathcal{F}}=\left.\partial_{\tau} \mathcal{F}\left(\tau, \Gamma_{\tau}, \vec{x}+\tau \vec{\psi}\right)\right|_{\tau=0} .
$$

Proof. We only outline the proof here for completeness, and refer to $[34,43]$ for more details and background. A classical formula gives the following derivative for an integral on the manifold $\Gamma_{\tau}$

$$
\left.\frac{d J_{\tau}}{d \tau}\right|_{\tau=0}=\int_{\Gamma_{0}}\left(\dot{\mathcal{F}}+\mathcal{F} \operatorname{div}_{\Gamma} \vec{\psi}\right) d \Gamma,
$$

where $\operatorname{div}_{\Gamma}$ denotes the tangential divergence. Then we use Green's theorem in this manifold setting, namely,

$$
\int_{\Gamma_{0}}\left(\vec{\nabla}_{\Gamma} \mathcal{F} \cdot \vec{\psi}+\mathcal{F} \operatorname{div}_{\Gamma} \vec{\psi}\right) d \Gamma=\int_{\Gamma_{0}} \kappa \mathcal{F} \vec{\psi} \cdot \vec{n} d \Gamma
$$

valid here since the contour is assumed to be closed. Combining the previous two equations we infer (.1).

Note that $\dot{\mathcal{F}}-\vec{\nabla}_{\Gamma} \mathcal{F} \cdot \vec{\psi}$ can be interpreted as the particle derivative in the direction normal to the contour. 